\title{
On Effective Computation of Fundamental Units. II
}

\author{
By Michael Pohst, Peter Weiler and Hans Zassenhaus
}

\begin{abstract}
The new method for efficient computation of fundamental units of an algebraic number field originated by two of the authors in part $I$ is used to develop a powerful computer program. Besides the description of this program part II contains a complete list of fundamental units of algebraic number fields of degree $n=3,4,5,6$ and small absolute discriminant.
\end{abstract}

Introduction. In part I of this paper we described the theoretical improvements of our method of computing fundamental units in algebraic number fields which we achieved in the last few years. Those improvements were of considerable influence on the corresponding computer program. Since its first 1976 [2] implementation it was completely rewritten and changed in so many details that a new presentation cannot be avoided.

In Sections 1 and 2 of this paper we describe the applications of part I [6] to the algorithm for constructing fundamental units. Section 3 contains a complete list of numerical examples concerning algebraic number fields of small degree and small absolute discriminant. Besides the fundamental units the tables contain much information about fields of degree five and six which was so far unknown. The determination of those fields (and their subfields) is described in [3]. Besides the fundamental units we also listed the order of the torsion subgroup $T U_{F}$ of the unit group $U$, whenever it is different from 2. It was computed by the methods of Section 2 of [5].

All computations were carried out on the Control Data Cyber 76 of the Computer Center of the University of Cologne.

1. Input Data and Initialization. We consider algebraic number fields $F$ which are given by their degree $n$ over the rational number field $\mathbf{Q}$ and a monic irreducible polynomial

$$
f(x)=x^{n}+a_{1} x^{n-1}+\cdots+a_{n} \in \mathbf{Z}[x]
$$

such that $F=\mathbf{Q}(\rho)$ for a root $\rho$ of $f$. It is convenient to provide an integral basis $\omega_{1}, \ldots, \omega_{n}$ of $F$ for the input. (Integral bases can be computed by the methods in [1], for example.) Hence we assume that we know a matrix $M \in \mathbf{Z}^{n \times n}$ and a positive integer $m$ such that

$$
\left(\omega_{1}, \ldots, \omega_{n}\right)=\frac{1}{m}\left(1, \rho, \ldots, \rho^{n-1}\right) M^{t}
$$

Received April 14, 1981.

1980 Mathematics Subject Classification. Primary 12A45. 
is an integral basis of $F$. For the second step of the algorithm, the computation of fundamental units from a maximal set of independent ones, we need a lower bound $R_{L}$ for the regulator $R_{F}$ of $F$. The number $R_{L}$ is the only floating-point number among the input data.

After the input is read we carry out some calculations relating to $F$ which need to be done only once. Here and in the sequel we apply both fixed-point and floating-point arithmetics. Though it would be adequate for the problem to work only with integers this is not convenient and even slows down computations. We give a few reasons for this phenomenon.

In the course of the computations it is necessary to use rational numbers, for example, in solving systems of equations. But the appropriate arithmetic for rational numbers can hardly be realized on most computers because of bounded word-size. The implementation of higher level languages like Scratchpad or MACSYMA, where the numbers are allowed to be arbitrarily large, strongly depends on the type of the computer. Usually the facilities for using higher level languages are very limited.

The main algorithm produces lots of numbers of bounded absolute value of the norm. Therefore the method to compute those values should be as fast as possible. Integral computation methods require the determination of the right regular representation matrix and the computation of its determinant. Besides the remarks above the evaluation of a determinant costs $O\left(n^{3}\right)$ operations. But computing the conjugates $\omega_{i}^{(j)}$ as complex numbers instead, we need only $O\left(n^{2}\right)$ operations for the computation of a norm. Of course we have to take care of round-off errors. An error analysis will be given below.

Since we did our computations on a CDC-machine, we have a further reason to use floating-point arithmetic. There are not any hardware commands for integer multiplication or division. Hence it is only natural to do without.

All integral calculations are concerned with the construction of a multiplication table for the integral basis $\omega_{1}, \ldots, \omega_{n}$. For this purpose we apply elementary unimodular transformations to $M$ from the right to obtain $M$ in Hermite normal form, i.e., we obtain a new integral basis $\omega_{1}, \ldots, \omega_{n}$, where the entries $m_{i j}$ of $M$ now satisfy

$$
\begin{aligned}
& m_{i j}=0 \quad \text { for } 1 \leqslant i<j \leqslant n \\
& m_{j j}>0, \quad 0 \leqslant m_{j i}<m_{j j} \leqslant m \text { for } j=1, \ldots, n, i=1, \ldots, j-1 .
\end{aligned}
$$

Next we must reduce powers $\rho^{k}$ for $k \geqslant n$ by $(1.1)$, i.e., $f(\rho)=0$, leading to $R \in \mathbf{Z}^{(n-1) \times n}$ defined by

$$
\begin{aligned}
\rho^{i-1} \rho^{j-1}=\sum_{k=1}^{n} R(i+j-n-1, k) \rho^{k} \\
\quad(i+j-2=n, n+1, \ldots, 2 n-2) .
\end{aligned}
$$

From (1.4) we easily derive a multiplication table for products of basis elements $\omega_{i}$, $\omega_{j}$ : 


$$
\begin{aligned}
\omega_{i} \omega_{j} & =\left(\frac{1}{m} \sum_{\mu=1}^{i} m_{i \mu} \rho^{\mu-1}\right)\left(\frac{1}{m} \sum_{\nu=1}^{j} m_{j \nu} \rho^{\nu-1}\right) \\
& =m^{-2} \sum_{\mu=1}^{i} \sum_{\nu=1}^{j} m_{i \mu} m_{j \nu} \rho^{\mu-1} \rho^{\nu-1} \\
& =m^{-2} \sum_{k=1}^{n} s_{i, j, k} \rho^{k-1} \quad(\text { by }(1.4)) ;
\end{aligned}
$$

now the multiplication constants $S(i, j, k)$ of $\omega_{i} \omega_{j}=\sum_{k=1}^{n} S(i, j, k) \omega_{k}$ are recursively obtained for $k=n, n-1, \ldots, 1$ by

$$
S(i, j, k)=\left(\frac{1}{m} s_{i, j, k}-\sum_{\nu=k+1}^{n} S(i, j, \nu) m_{\nu k}\right) / m_{k k} \quad(i, j=1, \ldots, n) .
$$

For floating-point calculations first of all we need to compute all zeros $\rho=$ $\rho_{1}, \ldots, \rho_{n}$ of $f(x)$. We order them as usual such that

$$
\rho_{1}, \ldots, \rho_{r_{1}} \in \mathbf{R} ; \quad \rho_{r_{1}+1}, \ldots, \rho_{n} \in \mathbf{C} \backslash \mathbf{R}, \quad \bar{\rho}_{r_{1}+i}=\rho_{r_{1}+r_{2}+i} \quad\left(1 \leqslant i \leqslant r_{2}\right) .
$$

The computation is done by the ZPOLR routine of the IMSL library which is available at most computer centers. The routine yields the zeros up to single machine precision, in our case 14 digits. Also we obtain the rank $r$ of the unit group $U_{F}$ of $F$,

$$
r=r_{1}+r_{2}-1 \text {. }
$$

At the next step we compute the conjugates $\omega_{i}^{(j)}(i, j=1, \ldots, n)$ on an array $D \in \mathbf{R}^{n \times n}$ by (1.6) and (1.2).

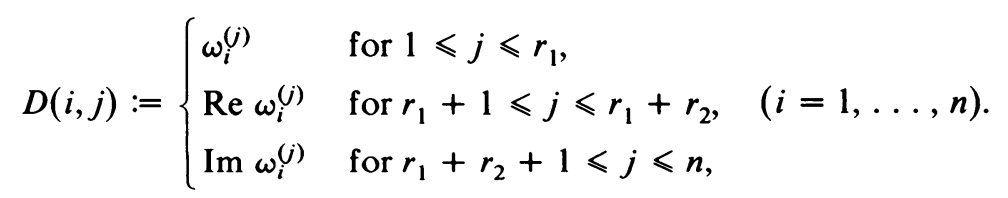

Then for any integer $x=\xi_{1} \omega_{1}+\cdots+\xi_{n} \omega_{n} \in o_{F}$ its absolute norm is easily calculated by means of

$$
\begin{aligned}
|N(x)|= & \left|\prod_{j=1}^{r_{1}}\left(\sum_{i=1}^{n} \xi_{i} D(i, j)\right)\right| \\
& \cdot \prod_{j=r_{1}+1}^{r_{1}+r_{2}}\left(\left(\sum_{i=1}^{n} \xi_{i} D(i, j)\right)^{2}+\left(\sum_{i=1}^{n} \xi_{i} D\left(i, j+r_{2}\right)\right)^{2}\right) .
\end{aligned}
$$

Since the right-hand side of (1.9) is computed in floating-point and $N(x)$ is an integer, we must make sure that the round-off error is less than 0.5 .

Since the zeros of $f$ are calculated up to single machine precision, we assume that they are affected by an error $\varepsilon$ which is less than $10^{-14}$ in our case. Hence we obtain an error for $D(i, j)$ of size

$$
\varepsilon \sum_{k=2}^{i}(k-1)\left|\rho_{j}\right|^{k-2} \quad(i \geqslant 2 ; j=1, \ldots, n) .
$$


If $x=\xi_{1} \omega_{1}+\cdots+\xi_{n} \omega_{n}$ is an integer of $F$, the error in the calculation of its conjugate $x^{(j)}$ will be at most

$$
\delta_{j}:=\varepsilon \sum_{i=2}^{n}\left|\xi_{i}\right| \sum_{k=2}^{i}(k-1)\left|\rho_{j}\right|^{k-2} \quad(j=1, \ldots, n) .
$$

Since all $x \in o_{F}$ whose norms we compute in this way stem from some transformation of the fundamental parallelotope $\pi,|N(x)|$ is bounded from above by a constant $U B$ which can easily be calculated ((1.3), (1.4) in [6]). Therefore the total error in computing $N(x)$ is bounded by

$$
\delta:=U B \sum_{j=1}^{n} \frac{\delta_{j}}{\left|x^{(j)}\right|} .
$$

For $\delta \leqslant 0.2$ the norm computation is certainly correct. For $\delta>0.2$ we either eliminate $x$, or-if we can expect $|N(x)|$ to be small-we compute $N(x)$ as the determinant of the right regular representation matrix of $x$.

2. The Algorithm. As described in part I of this paper [6] we construct integers $x \in o_{F}$ of bounded norm and try to extract units from them. We store integers of norms $|N(x)|>1$ into an array $V$, independent units into an array $V U$. Let $N O$ be the number of $x$ in $V, N U$ the number of units in $V U$. The initial values are $N O=N U=0$, of course. We now state the algorithm and give the necessary explanations below.

1. Algorithm for the Computation of Fundamental Units.

1. If all lattice points of the parallelotope under consideration are eliminated (see 2), make a linear transformation and compute the lattice points of the transformed parallelotope.

2. Take the next lattice point, eliminate it from the set under consideration and compute the corresponding integer $x \in o_{F}$ and $|N(x)|$.

3. Test $x$ with respect to $V$ and $V U$.

4. If $N U=r$, go to 5, else to 1 .

5. Compute a system of fundamental units from $V U(1), \ldots, V U(r)$.

While this presentation gives an overall plan of the algorithm, we need be more specific in regard to most of its steps.

Step 1. Transformations of the fundamental parallelotope and the determination of the lattice points in the transformed parallelotope were discussed at length in [6]. The implementation of those methods on a computer only requires the computation of the Hermite normal form of a matrix and some elementary integral calculations.

Step 2. Methods for the computation of $|N(x)|$ were developed in Section 1 of this paper.

Step 3. This step is elementary again, but it requires a careful consideration of all possibilities which can occur. Hence we explain this step in greater detail. In case $x$ is a unit, we examine whether it is dependent on $V U(1), \ldots, V U(N U)$ (see [2]). If it is, eliminate $x$ and go to 1 . Otherwise set $N U:=N U+1, V U(N U):=x$, and go to 4 . In case $|N(x)|>1$, we proceed to check the multiplicative relation of $x$ with the elements of $V$. Let $k$ denote the index of the next element of $V$ to be tested. The initial value of $k$ is 1 . 
2. Algorithm for Testing $x$ if $|N(x)|>1$.

Input: Arrays $V, V 1$, number $N O$. Set $k=1, l=0$.

1. In case $k>N O$, go to 7 .

2. For $|N(V(k))|>|N(x)|$ go to 5 , in case of equality to 4 .

3. Test $N(x) / N(V(k)) \in \mathbf{Z}$. If it is not, go to 6. Else test $x / V(k) \in o_{F}$. If it is not, go to 6. Else set $x:=x / V(k), k:=1$ and go to 2 .

4. Test $x / V(k) \in o_{F}$. If it is not, go to 6. Else $\varepsilon:=x / V(k)$ is a unit. We check, whether $\varepsilon$ is independent of $V U(1), \ldots, V U(N U)$. If it is not, we go to 6. If it is, set $N U:=N U+1, V U(N U):=\varepsilon$. For $N U=r$ go to step 5 of Algorithm 1, else to step 1 of Algorithm 1.

5. Test $N(V(k)) / N(x) \in \mathbf{Z}$. If it is not, go to 6. Else test $V(k) / x \in o_{F}$. If it is not, go to 6. Else eliminate $V(k)$, set $N O:=N O-1, l:=l+1, V 1(l):=V(k) / x$ and go to 1 .

6. Set $k:=k+1$, and go to 1 .

7. Set $N O:=N O+1, V(N O):=x$. In case of $l=0$, go to step 1 of Algorithm 1. Else set $x:=V 1(l), l:=l-1, k:=1$ and go to 2 .

Though these tests for $x$ are lengthy, they provide elements of very small norm very fast. However, from our computational experience, it is recommendable to eliminate elements of large absolute norm ( $>1000)$. That elimination speeded up the algorithm quite a bit.

Besides the methods of Section 1 of this paper we need a routine for the calculation of the quotient $q$ of two integers of $o_{F}$. We compute the quotient by solving a linear system of equations for the coefficients of $q$ represented by the integral basis $\omega_{1}, \ldots, \omega_{n}$.

Remark. The more sophisticated method of Section 2 in [6] has not yet been incorporated into the program. We will use it in a forthcoming paper for the computation of the class number of $F$.

Step 4. Clear.

Step 5. This step was already discussed in [2], [5]. Better lower bounds for $\boldsymbol{R}_{\boldsymbol{F}}$ from [6] made the computations a lot faster.

We conclude this section by adding two remarks which helped us to cut down the computation time. As already outlined in [2], it is of advantage not to start with step 5 of Algorithm 1, until we are convinced that we have already obtained a system of fundamental units. Hence, having obtained $r$ independent units we still go on constructing new units, check, whether they belong to $\langle V U(1), \ldots, V U(r)\rangle$ and change $V U$, if necessary, to obtain a larger subgroup of the full unit group $U_{F}$. If $\langle V U(1), \ldots, V U(r)\rangle$ does not change any more for-let us say-ten new units, we assume that we are done and proceed to the verification part, i.e., step 5 .

The important role of the choice of the integral basis $\omega_{1}, \ldots, \omega_{n}$ was thoroughly discussed in part I of this paper [6]. So, we usually start with a Minkowski-reduced basis [4]. If the search for $r$ independent units is not successful in a reasonable number of transformations of the fundamental parallelotope, a change of the integral basis is recommended. Unfortunately we can hardly tell which basis will be optimal. As a rule of thumb we recommend to choose basis elements of small norm. Sometimes it is of advantage to make the largest conjugate of $\omega_{i}$ small 
( $i=n, n-1, \ldots, 1)$ by subtracting suitable multiples of $\omega_{i-1}, \ldots, \omega_{1}$. This procedure follows the construction in quadratic number fields. In $\mathbf{Q}(\sqrt{m}), m \equiv$ 2, 3 mod 4 square-free, for example, the basis $1,-[\sqrt{m}]+\sqrt{m}$ yields much smaller norms than the reduced one $1, \sqrt{m}$.

3. Tables of Fundamental Units for Fields of Degrees $n=3,4,5,6$ and Small Absolute Discriminants. Applying our method to realize the computer program description given above, we succeeded in listing the fundamental units of 192 fields of small degree and small absolute discriminants. For each degree $n=3,4,5,6$ and each reality behavior $r_{1}, r_{2}$ we fix an upper bound $T_{r_{1}, r_{2}}$ for $\left|d_{F}\right|$. Then the table contains all fields $F$ of corresponding type with $\left|d_{F}\right| \leqslant T_{r_{1}, r_{2}}$, each of them represented by precisely one equation up to field isomorphism. These results are new for $n=5,6$. They were obtained by the methods of [3]. The tables are set up as follows.

Column 1 gives the number of the field for the subcase under consideration.

Column 2 contains the field discriminant (by increasing order of magnitude of their absolute value).

Column 3 contains the coefficients $a_{(1)}, \ldots, a_{(n)}$ of a generating equation $f(x)=x^{n}$ $+a_{(1)} x^{n-1}+\cdots+a_{(n)}$ for $F$.

Column 4 contains a $\mathbf{Z}$-basis $\omega_{1}, \ldots, \omega_{n}$ of $o_{F}$, in case the power basis $1, \rho, \ldots, \rho^{n-1}$ for a root $\rho$ in $f$ generates a proper subring of $o_{F}$.

Column 5 contains a system of fundamental units of $F$. Each unit $\varepsilon$ is represented by its coefficients $e_{(1)}, \ldots, e_{(n)}$ with respect to $\omega_{1}, \ldots, \omega_{n}: \varepsilon=e_{(1)} \omega_{1}$ $+\cdots+e_{(n)} \omega_{n}$.

Column 6 contains the regulator of $F$ (up to 5 decimal places).

Column 7 contains all intermediary fields between $\mathbf{Q}$ and $F$. Quadratic subfields are given explicitly, in case of cubic subfields we list the corresponding entry of Table I (i.e. I.1.1, for example). Obviously column 7 needs to be used only in the cases $n=4,6$.

Column 8 contains the order of the torsion subgroup of the unit group $U$, if it is different from 2. Hence column 8 occurs only in subcase $n=4=2 r_{2}$ or $n=6=$ $2 r_{2}$.

Remark. The occurrence of units with relatively large coefficients $e_{(1)}, \ldots, e_{(n)}$ is due not to our choice of lattice points (which could have influenced the norm computation, see end of Section 1), but due to division of integers of $F$ as outlined in Algorithm 2 of Section 2. 


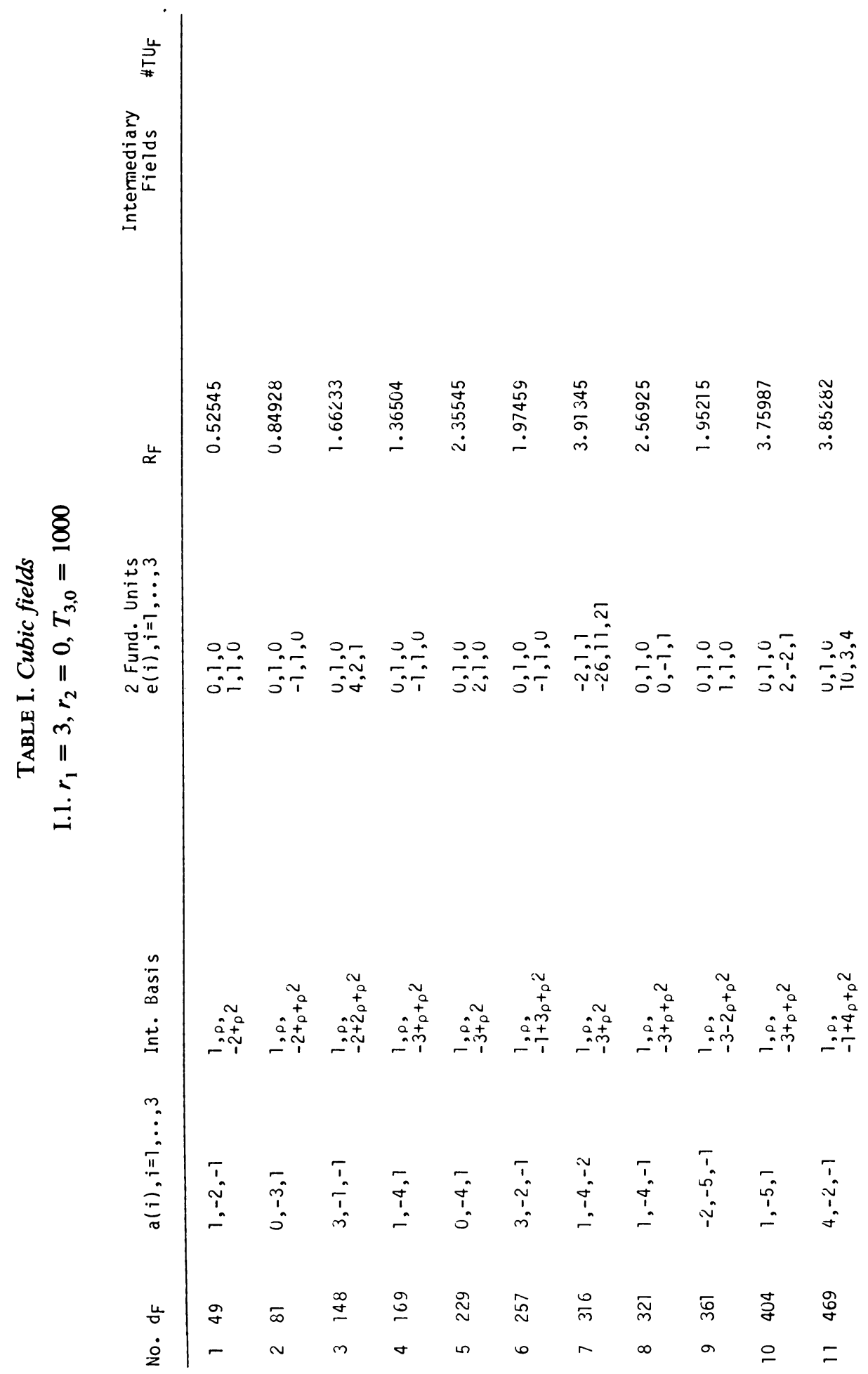




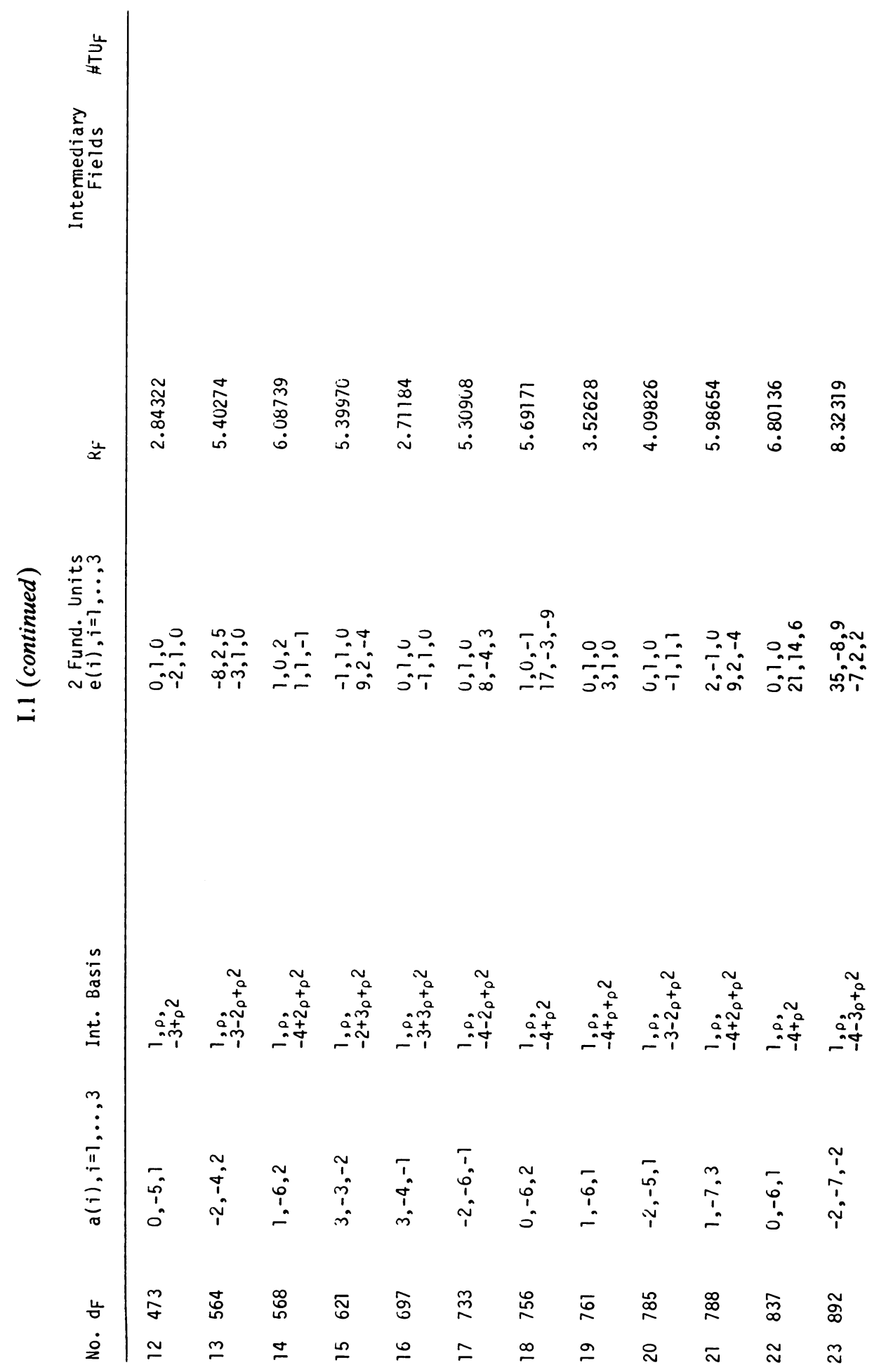




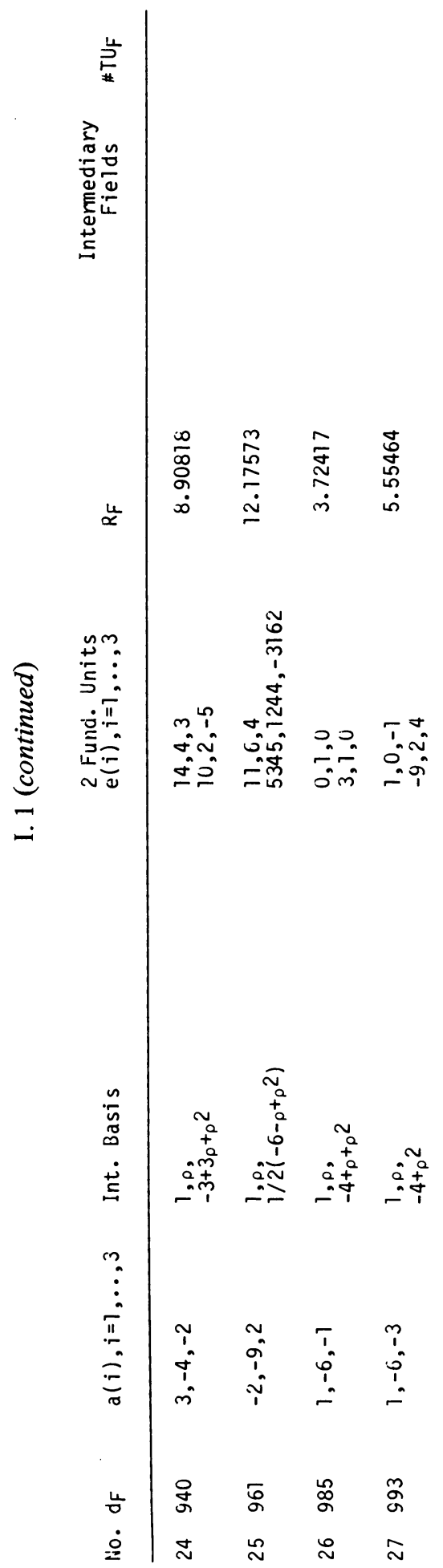




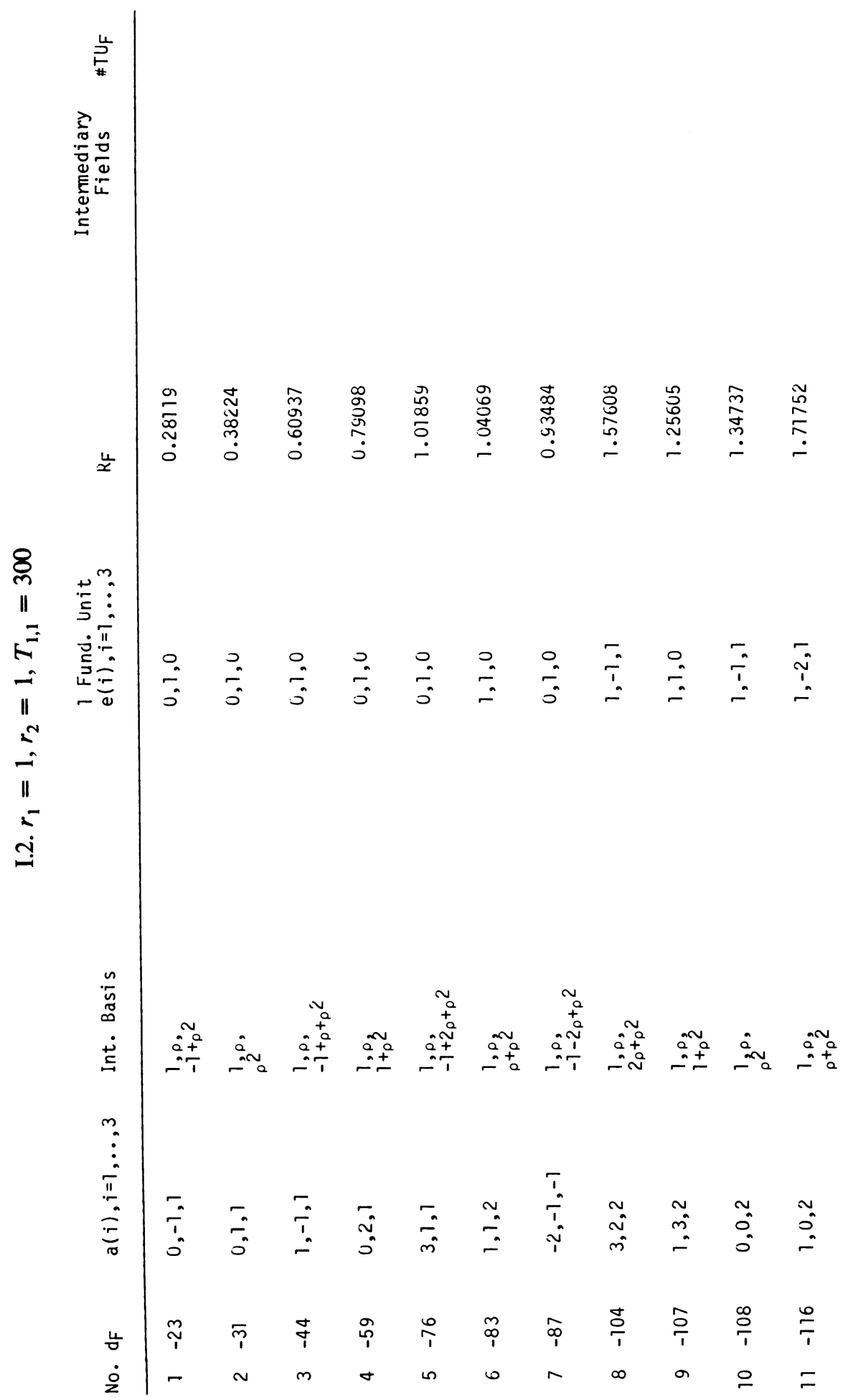


EFFECTIVE COMPUTATION OF FUNDAMENTAL UNITS. II

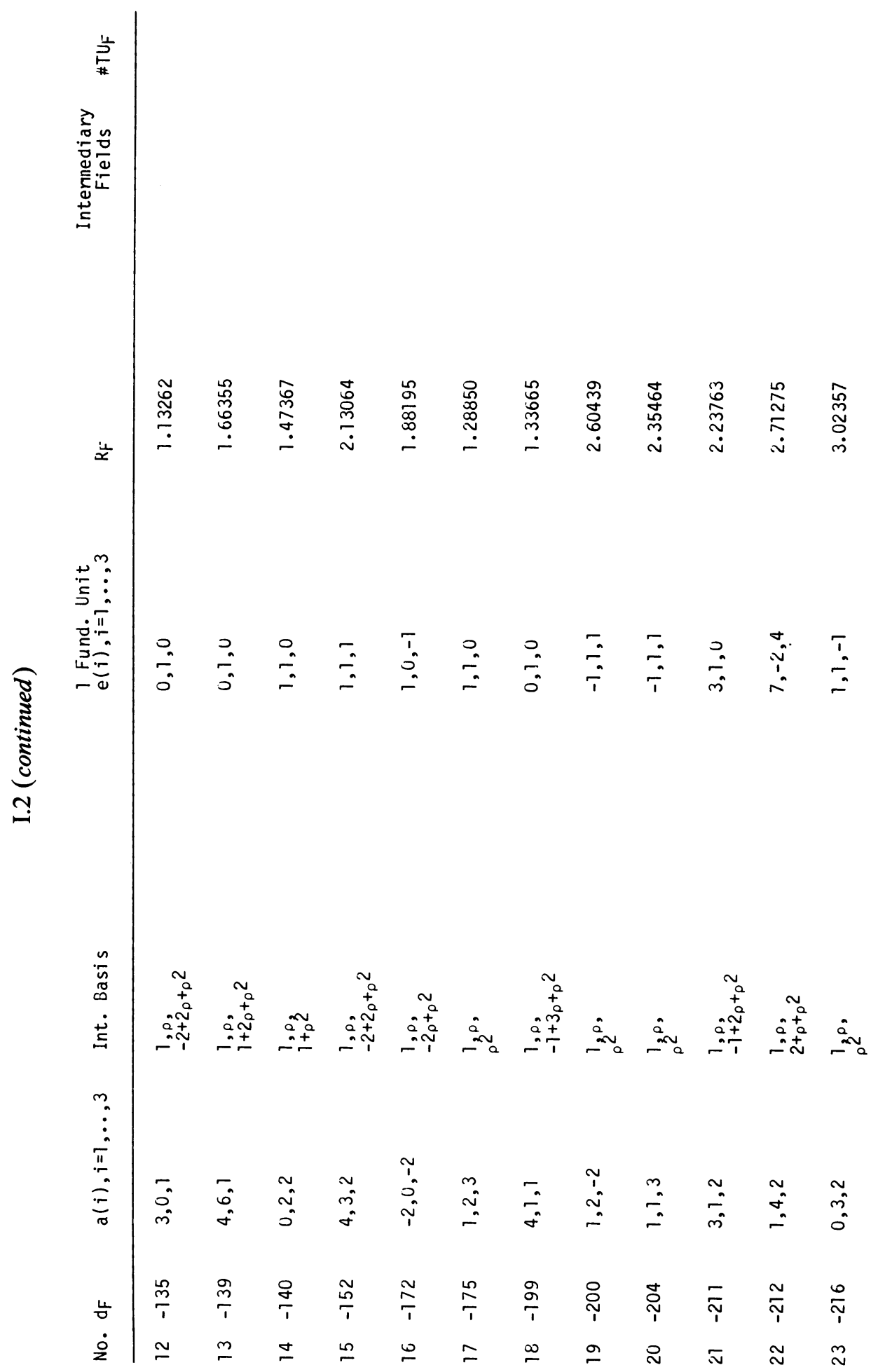




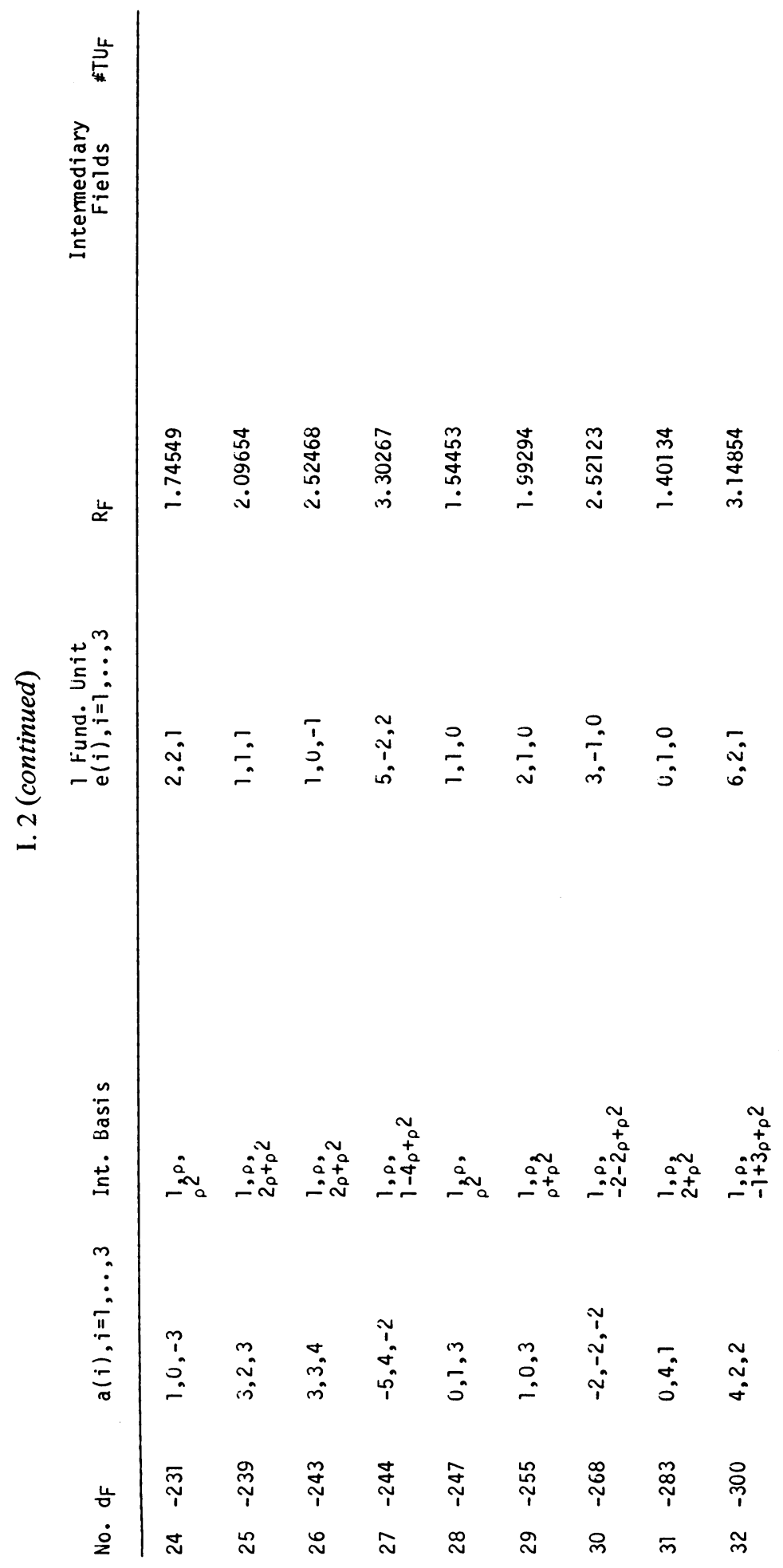




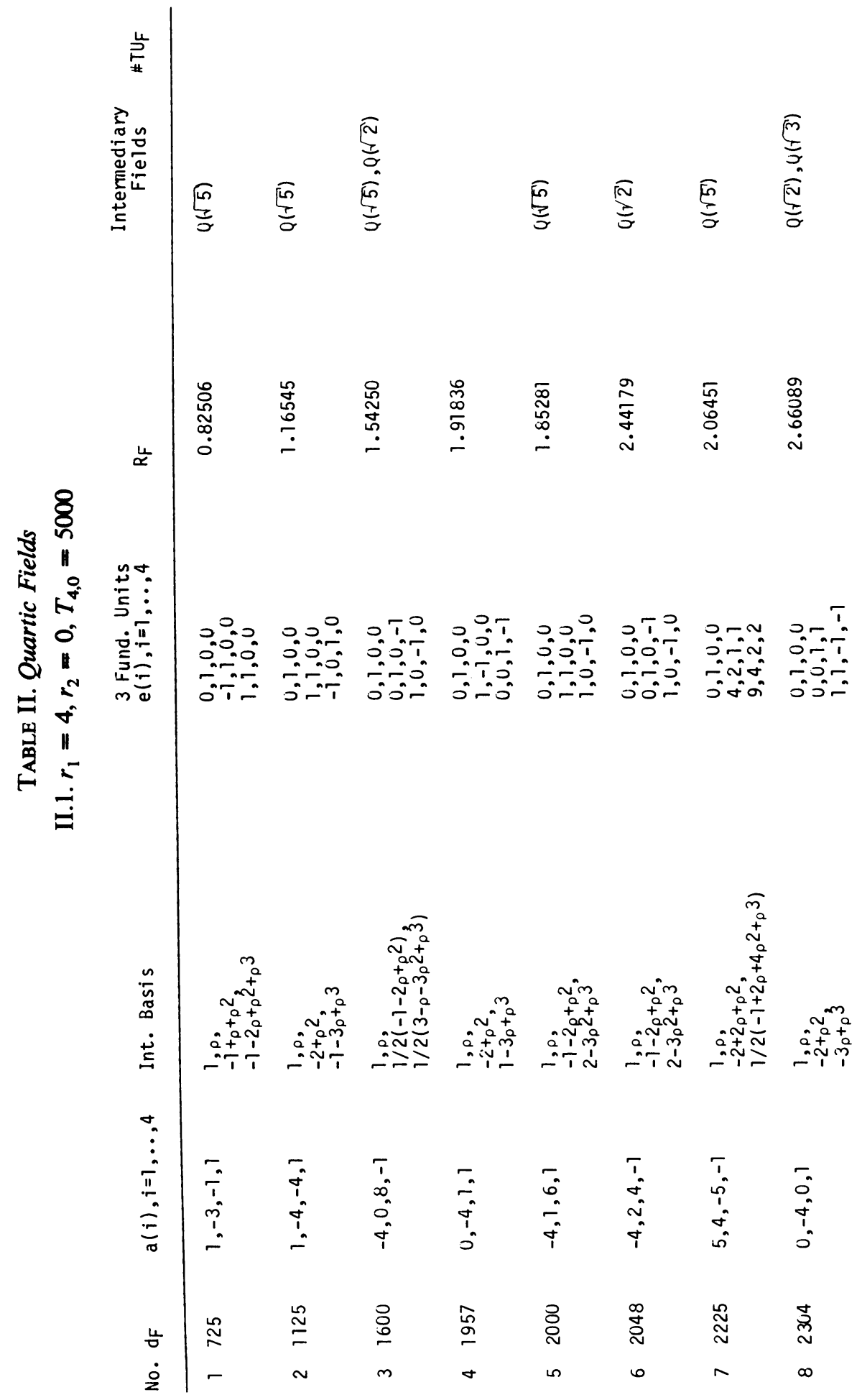




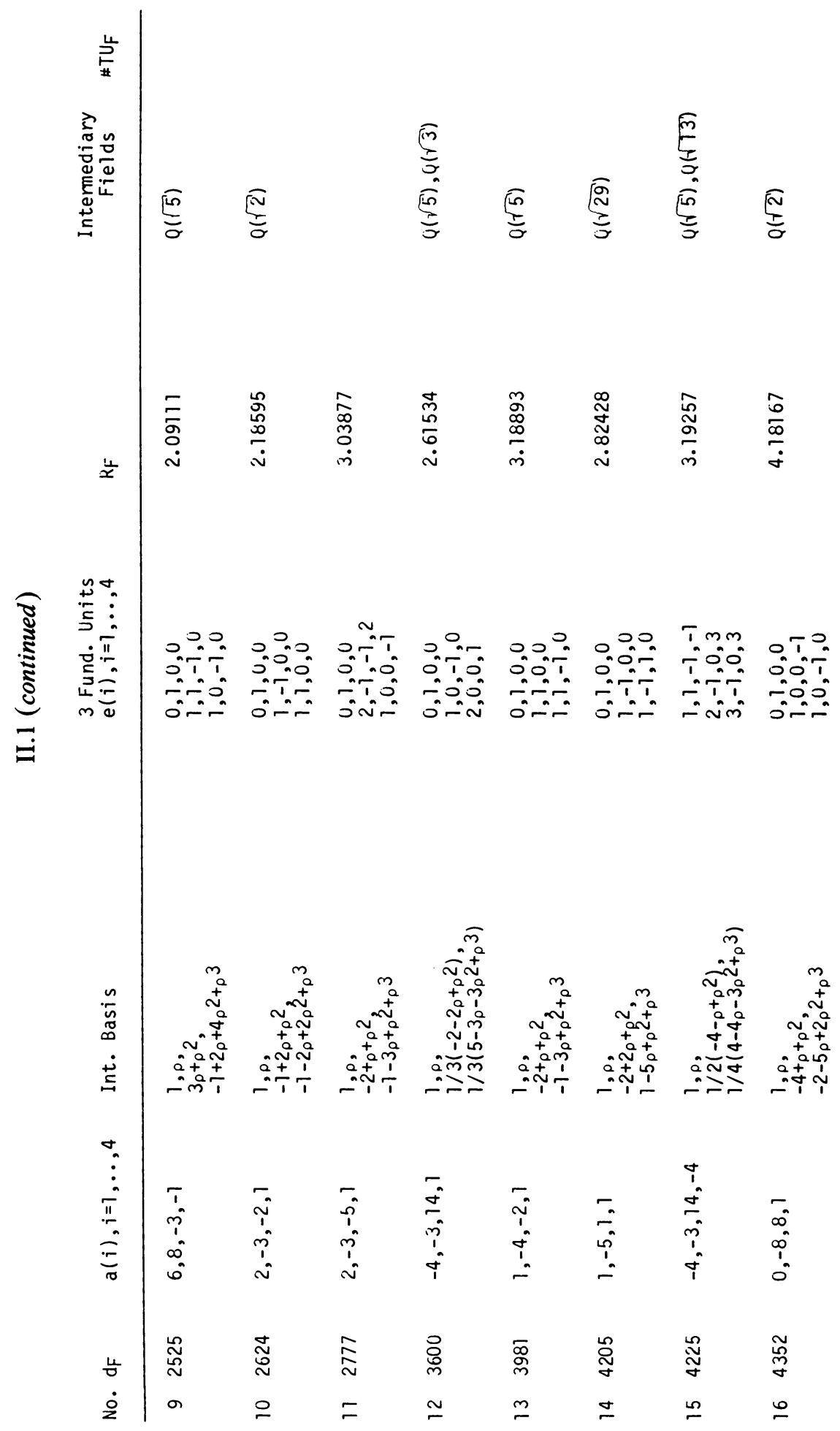




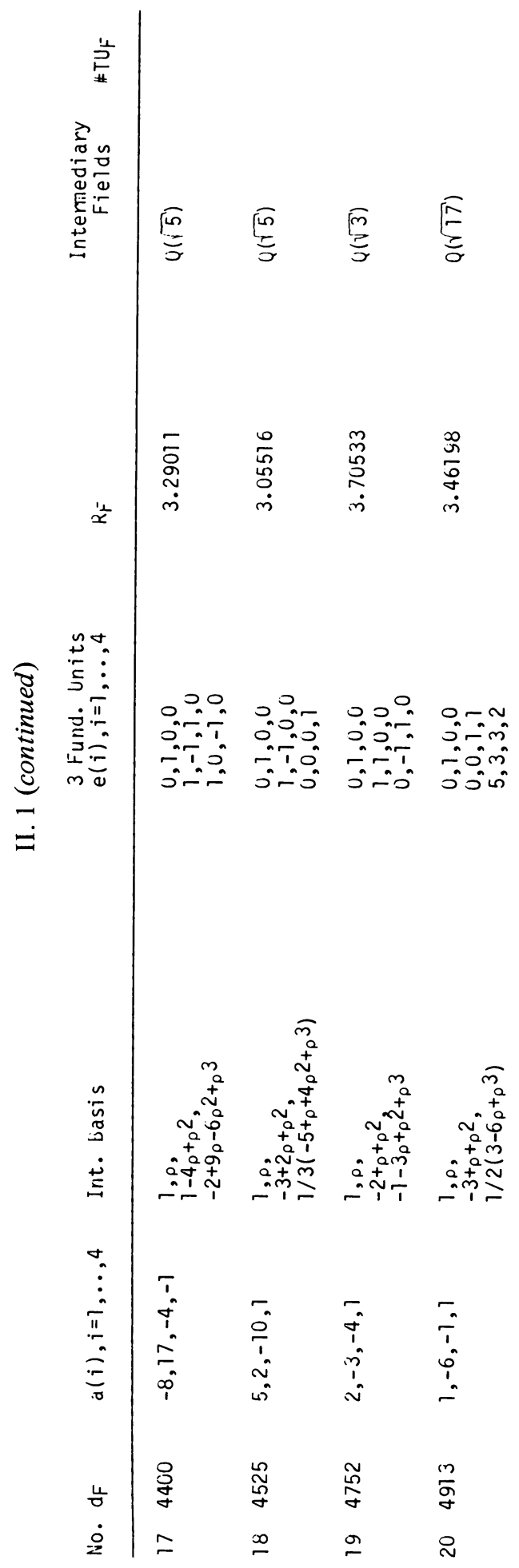




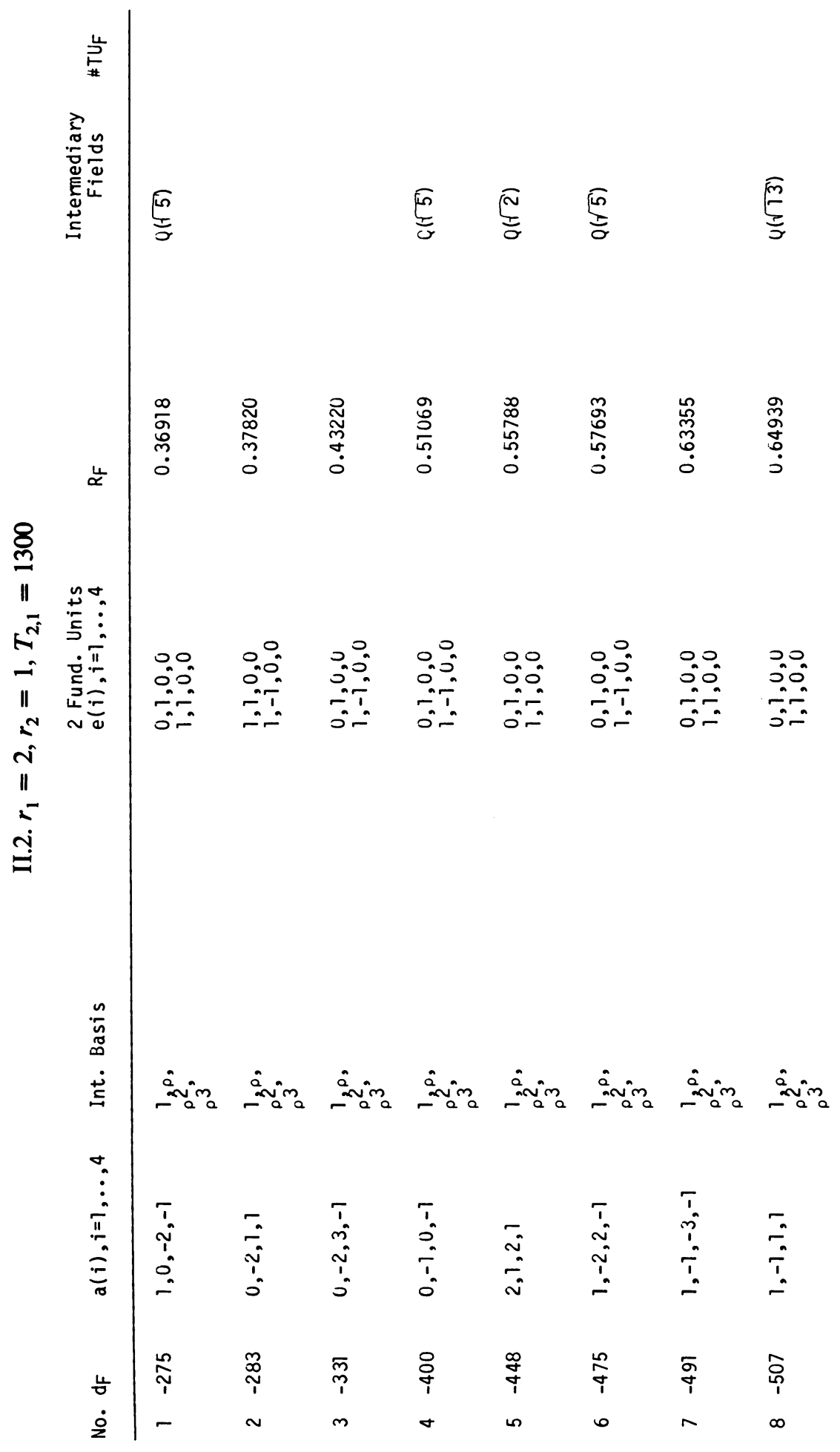




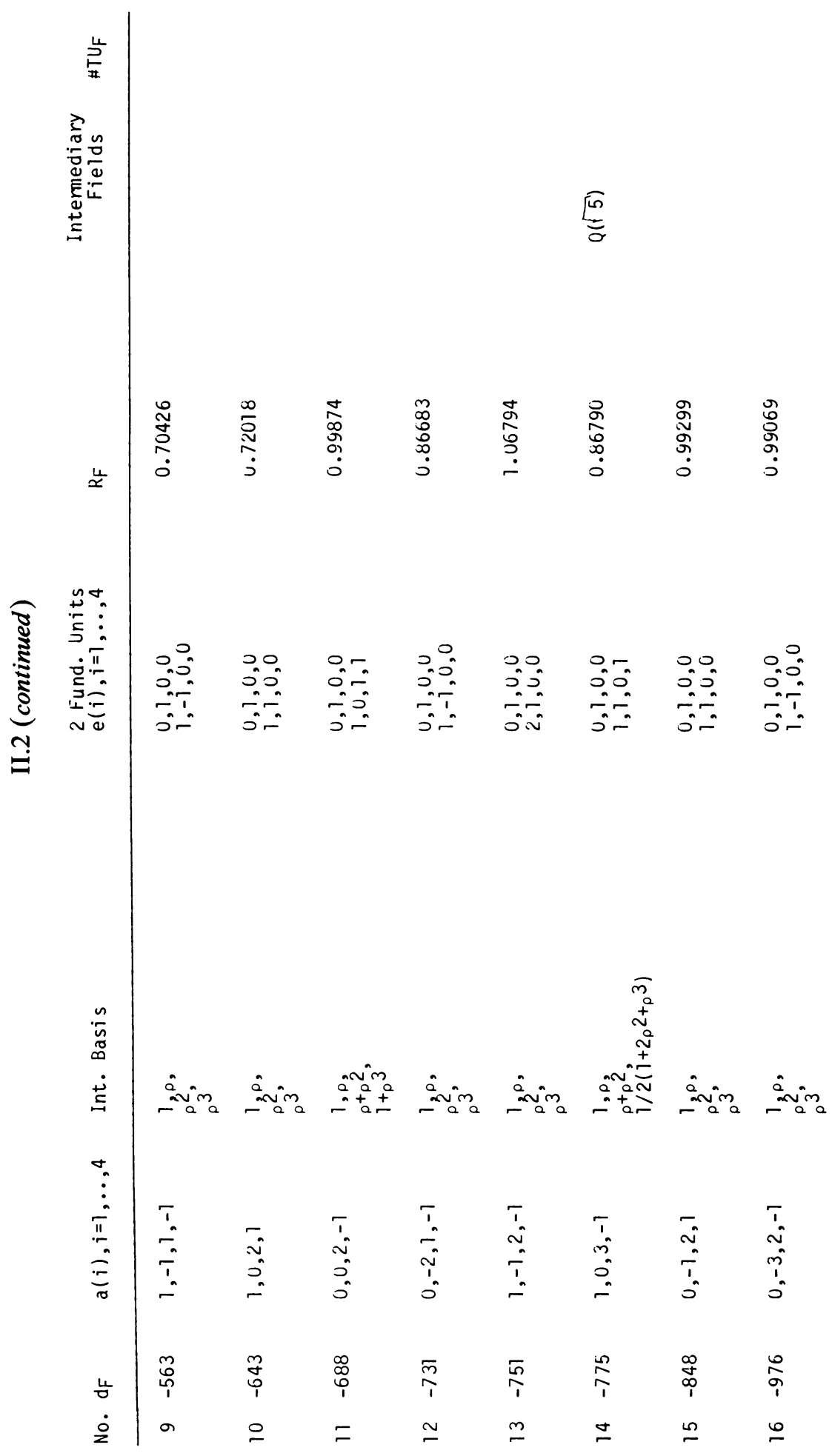




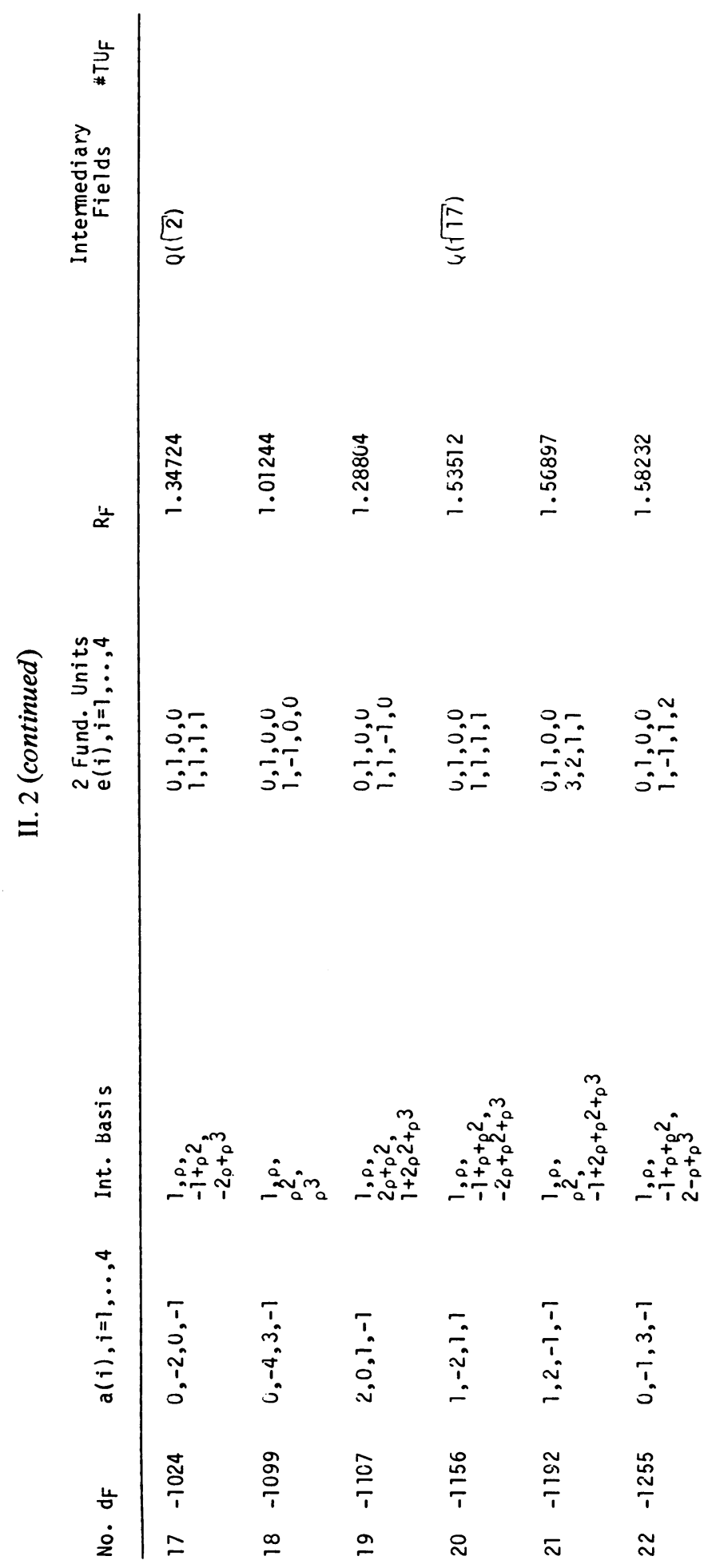


EFFECTIVE COMPUTATION OF FUNDAMENTAL UNITS. II

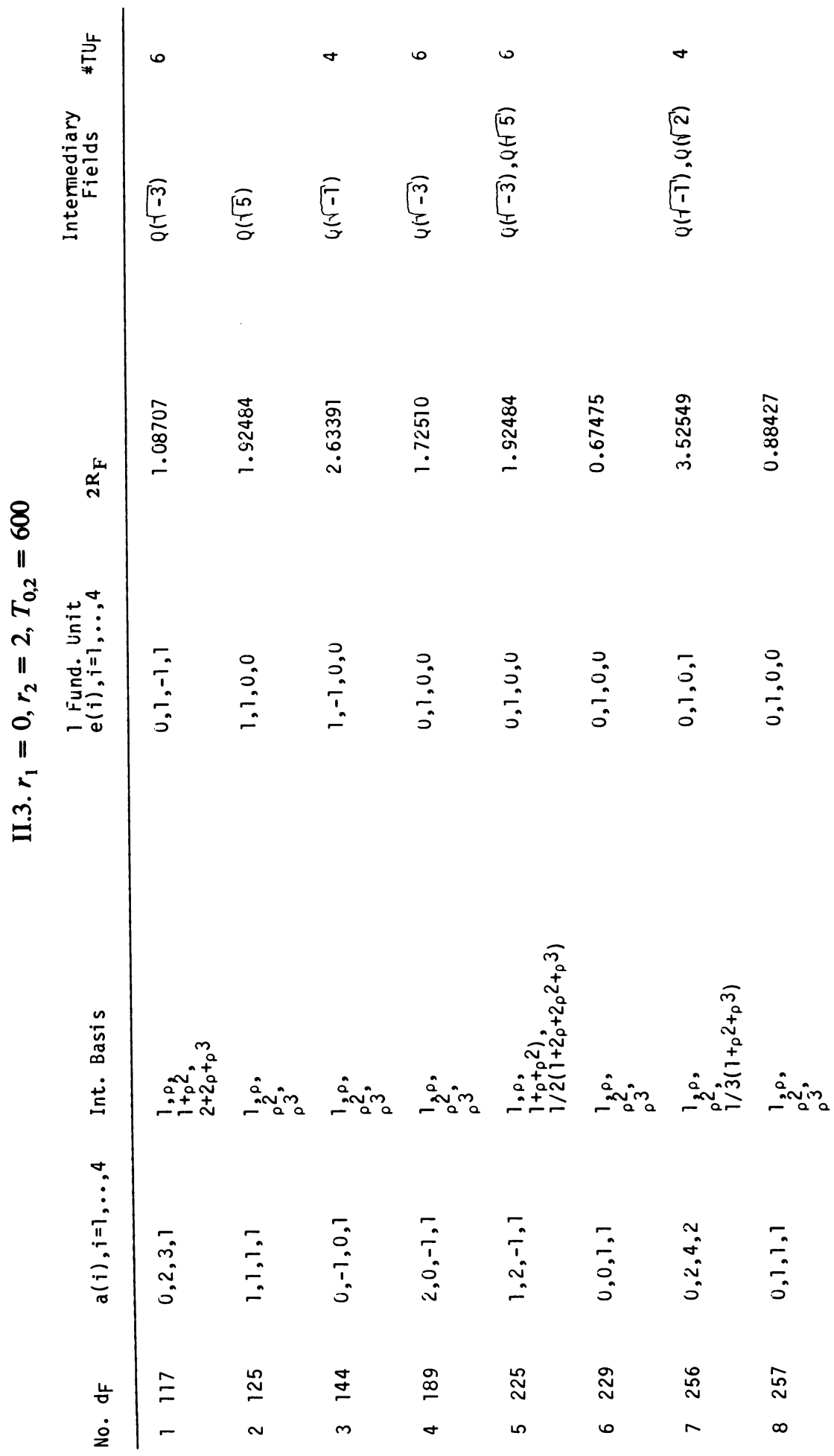




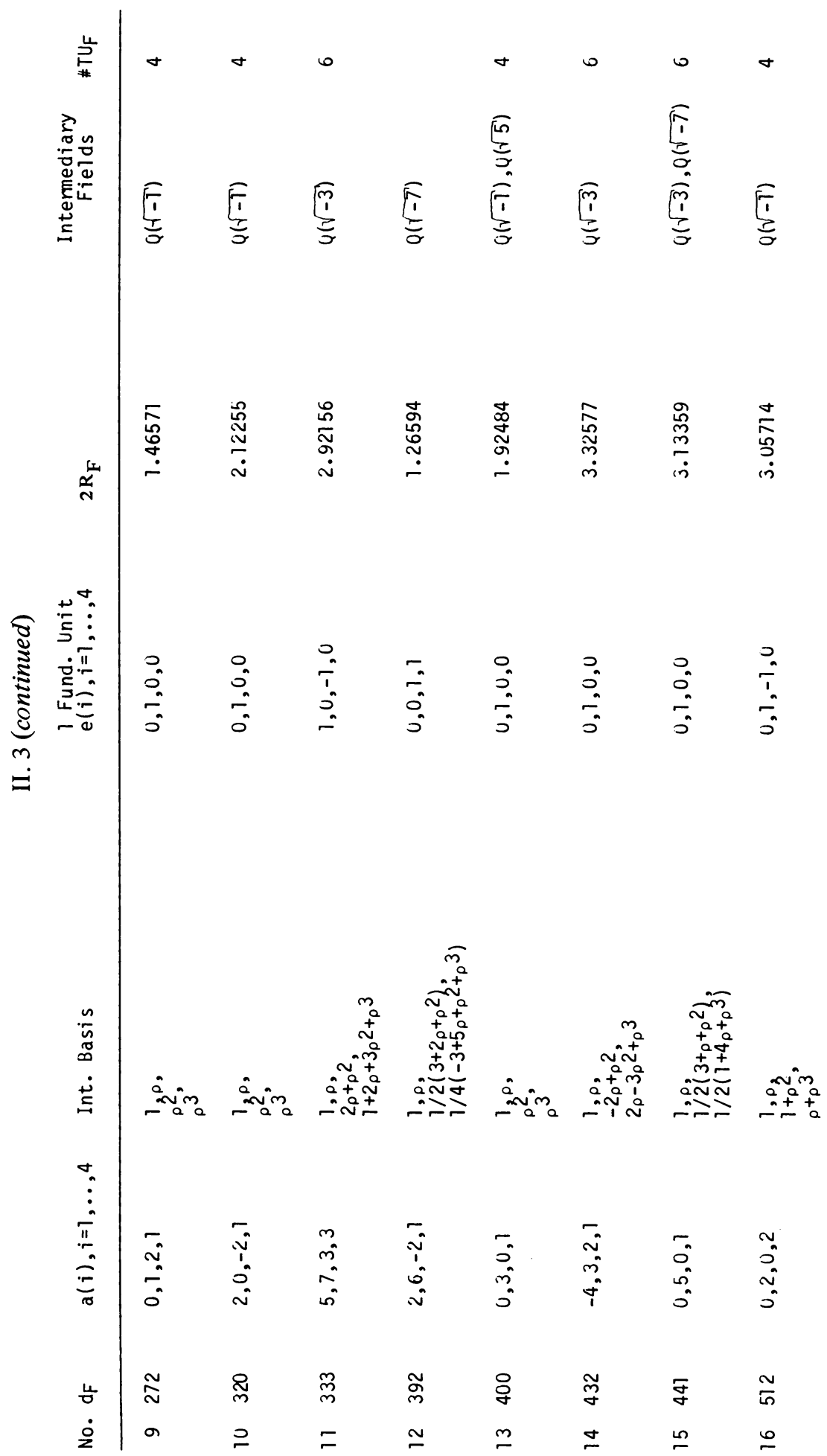




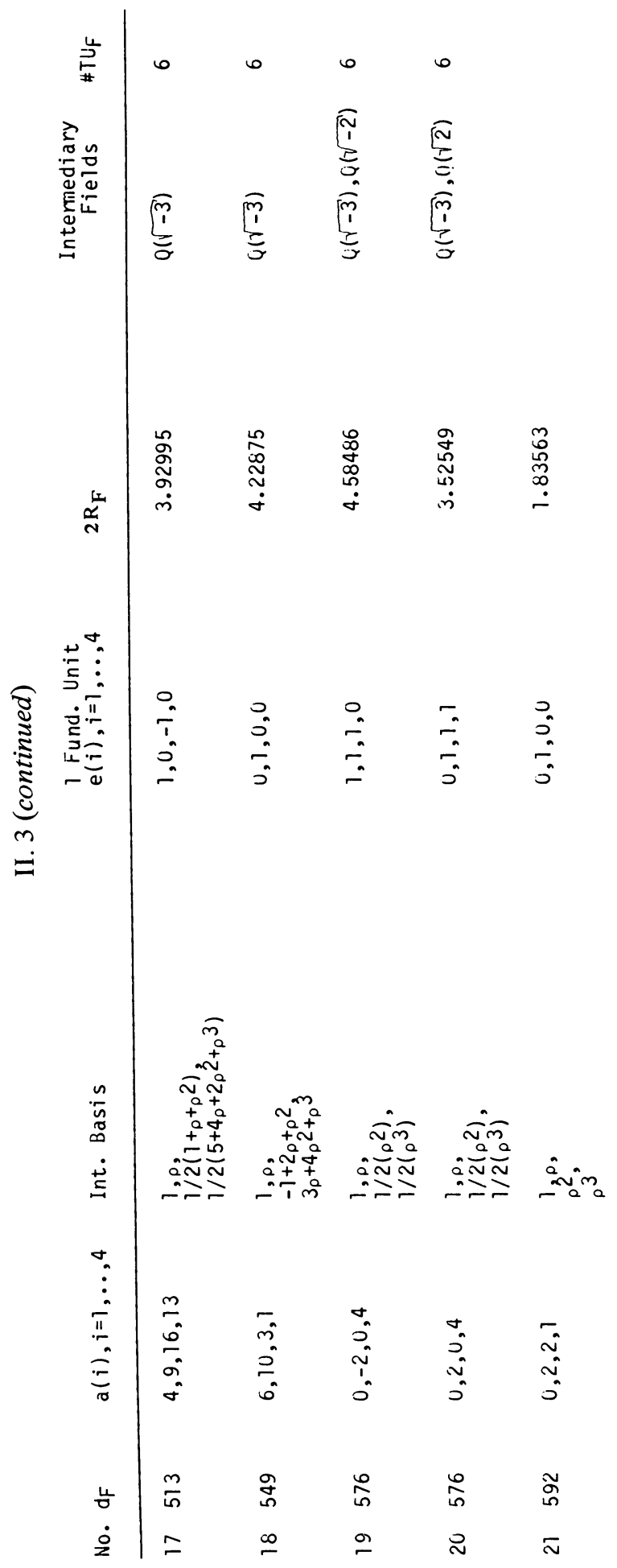




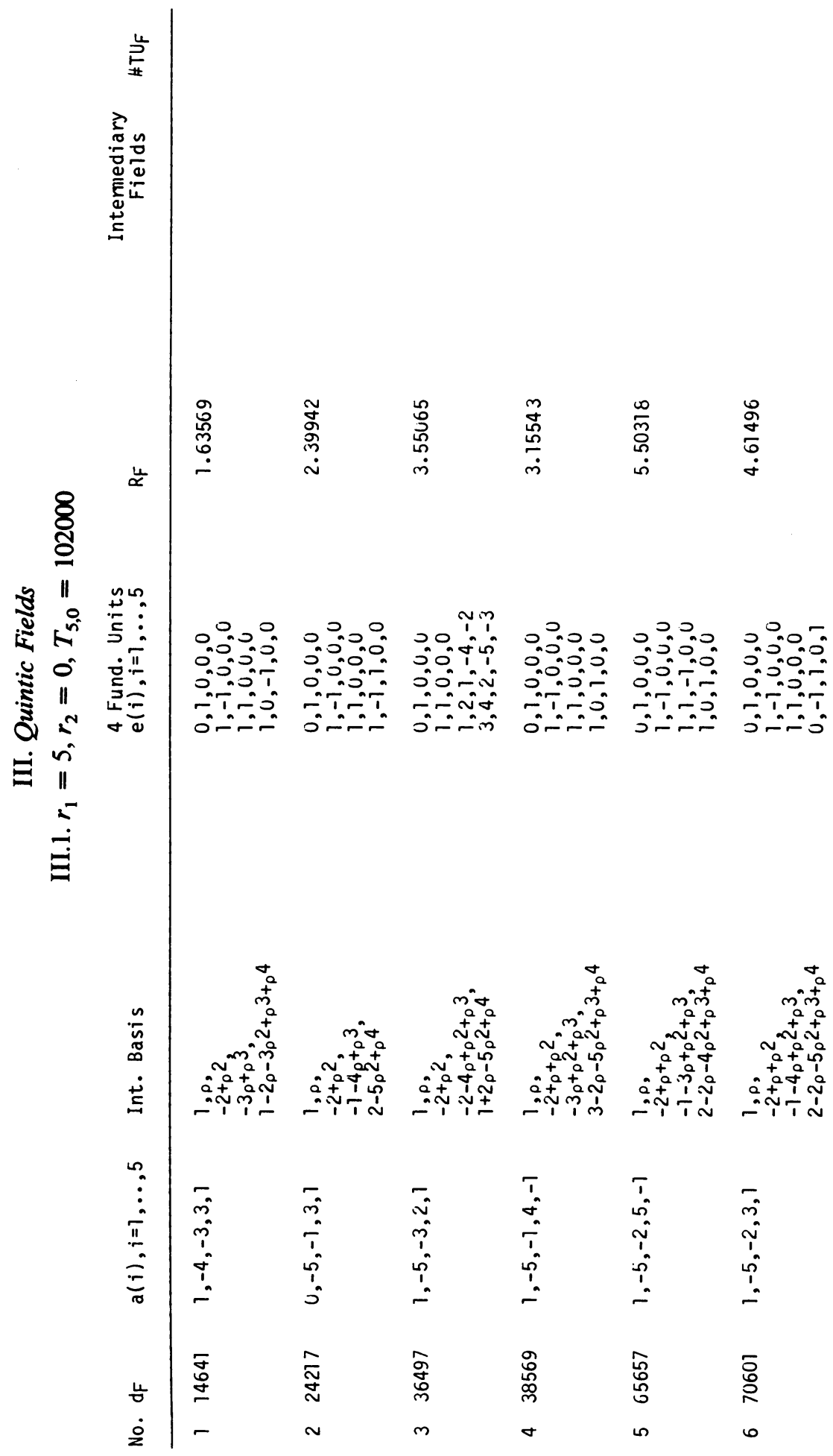




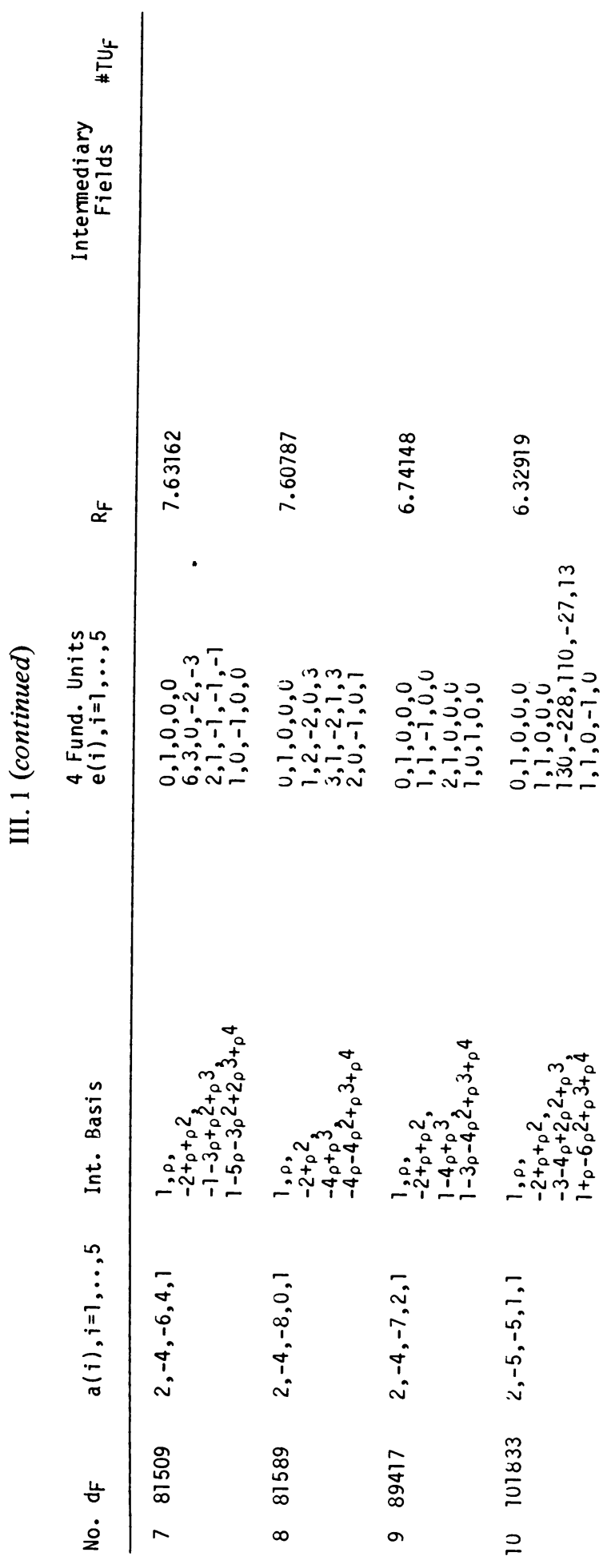




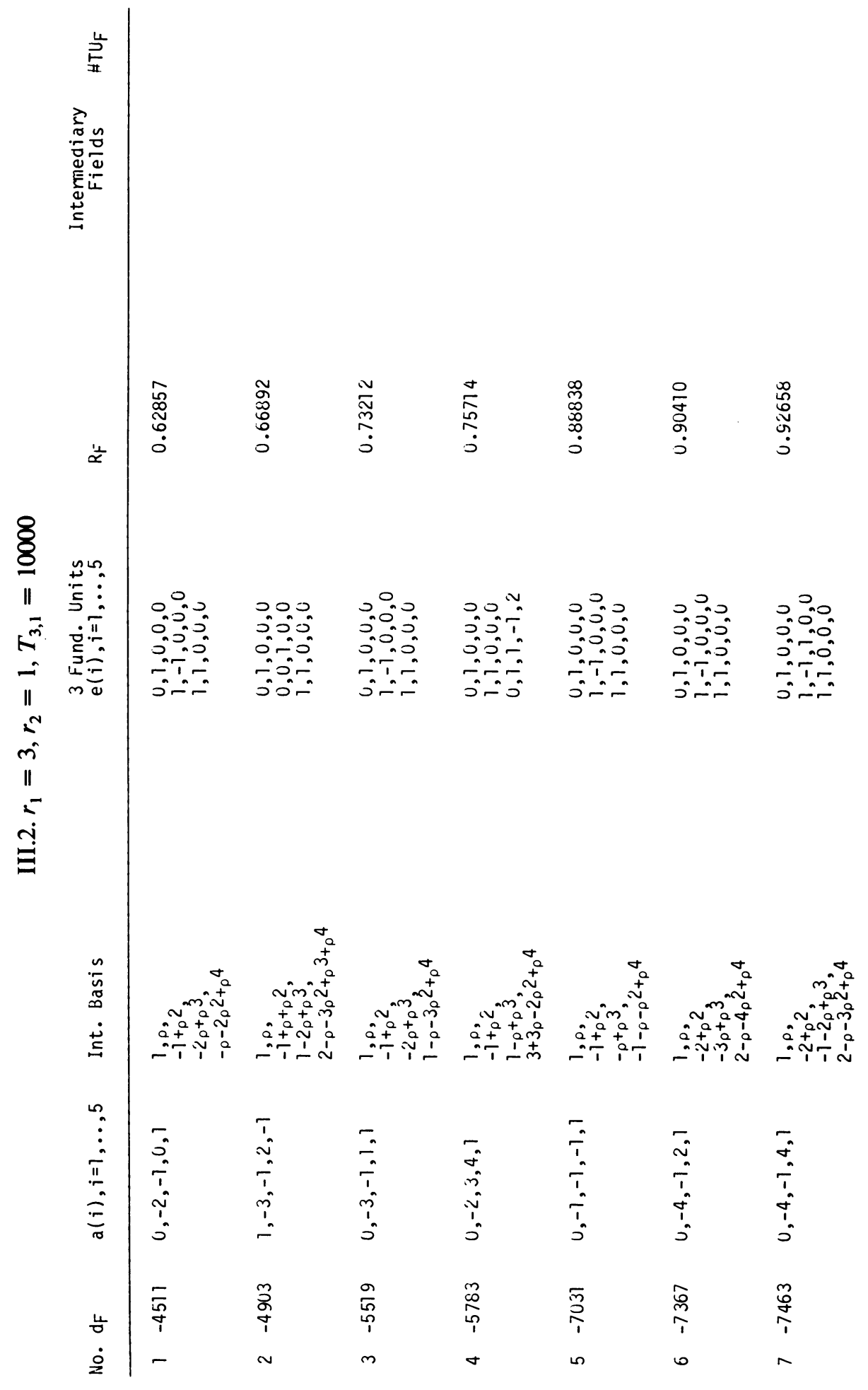




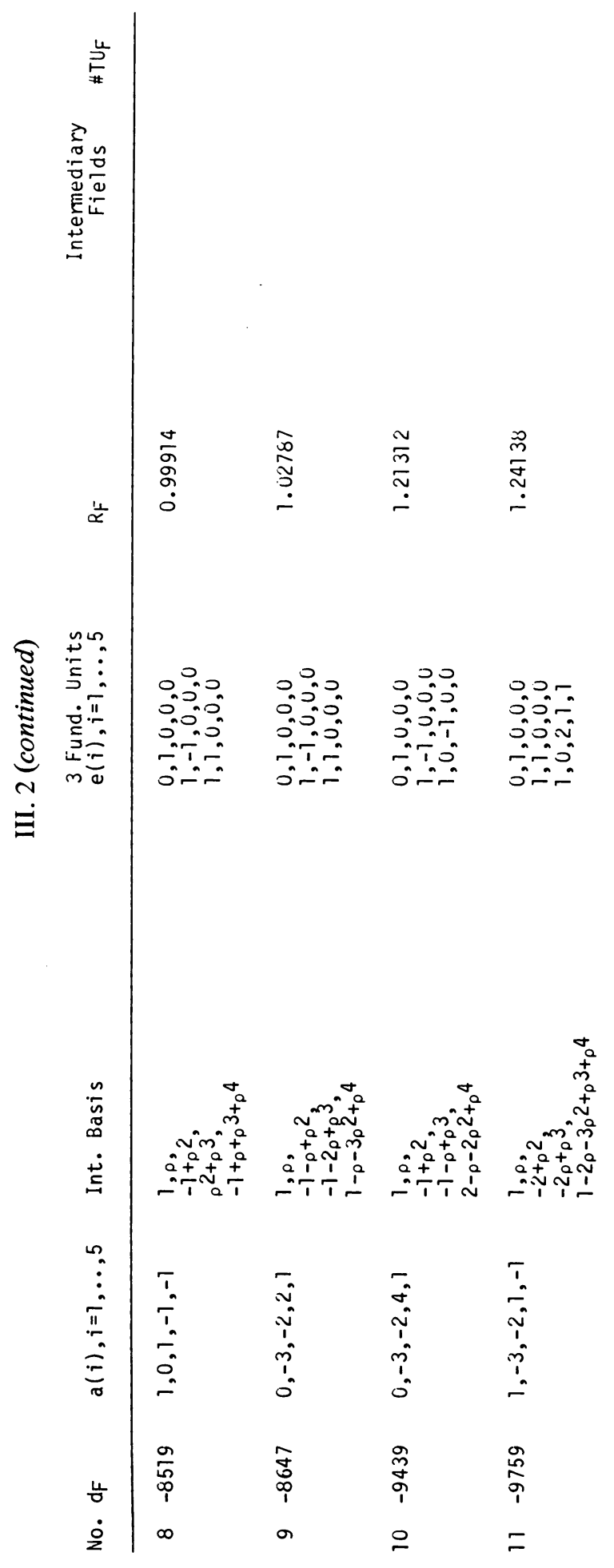




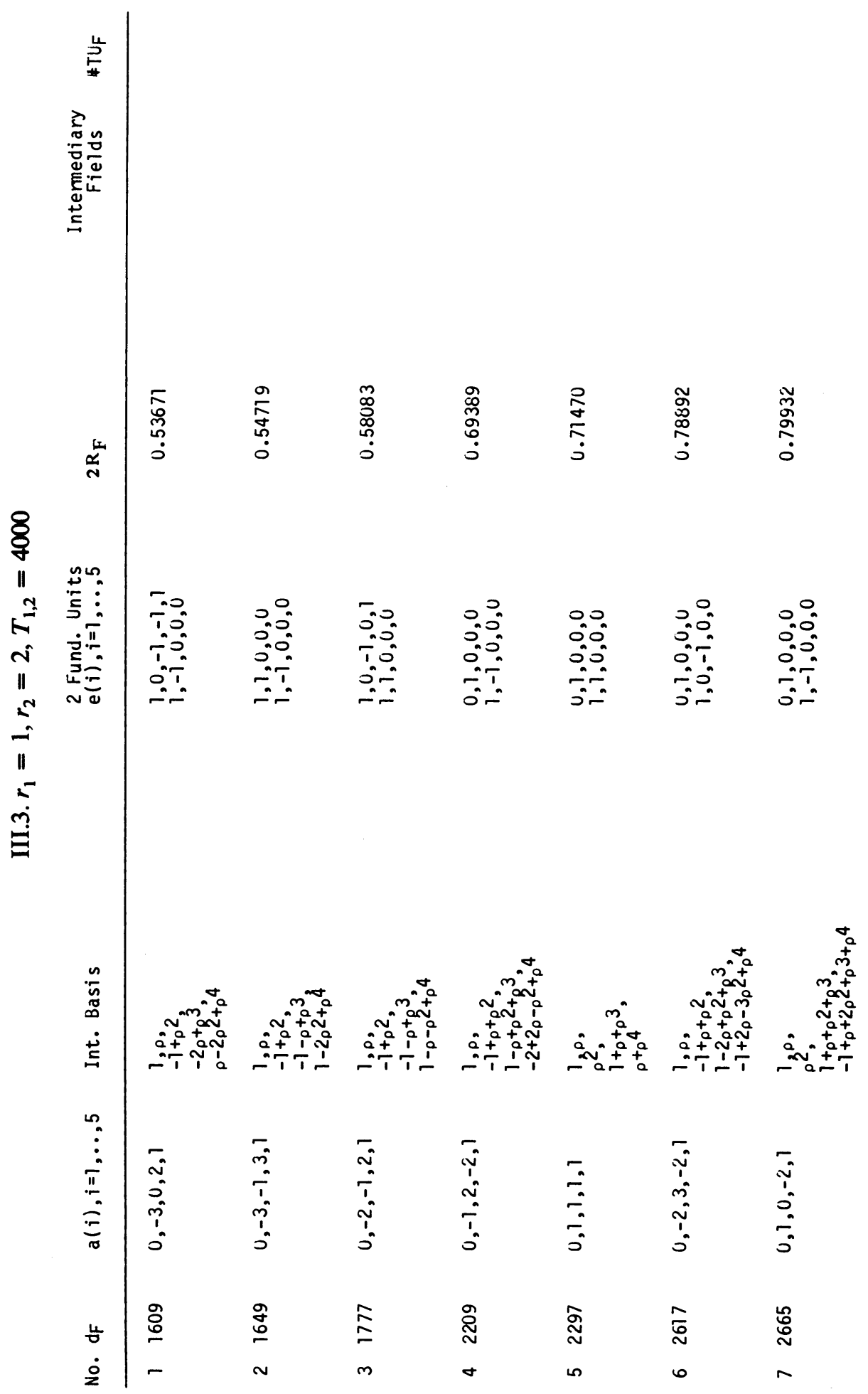




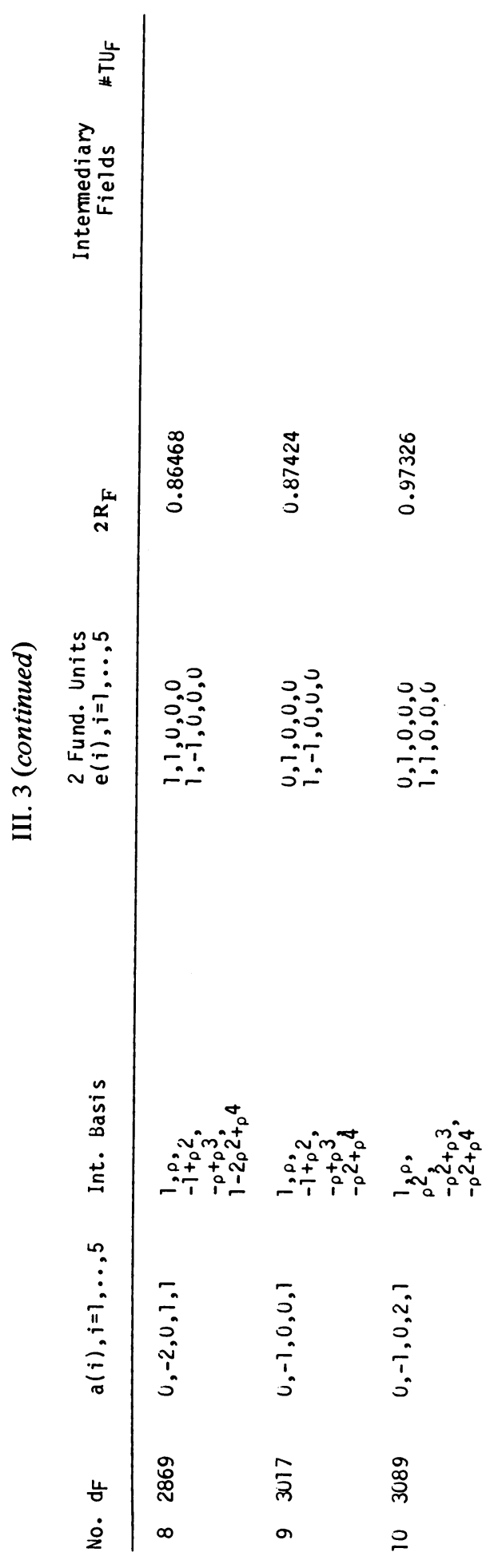




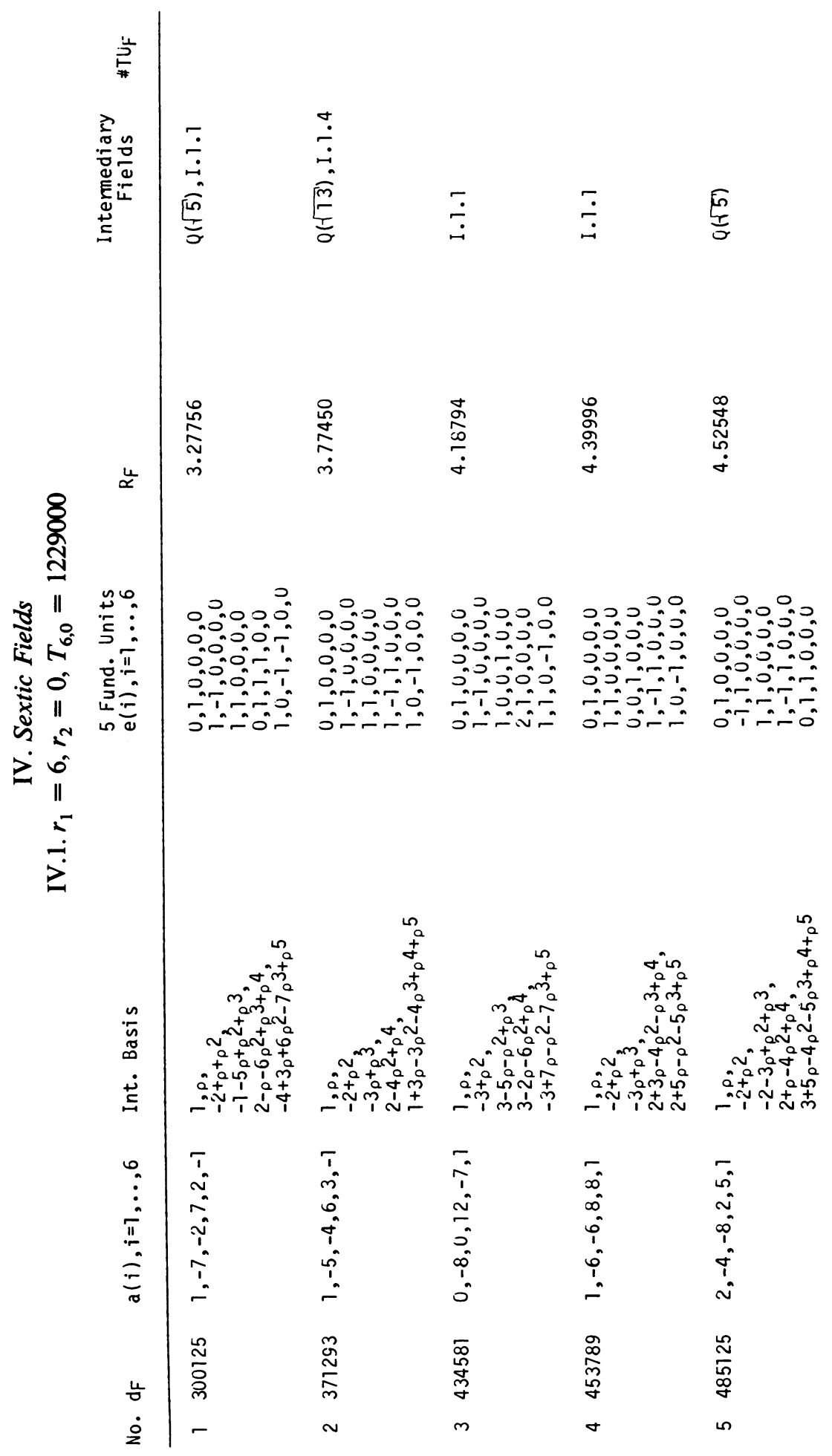




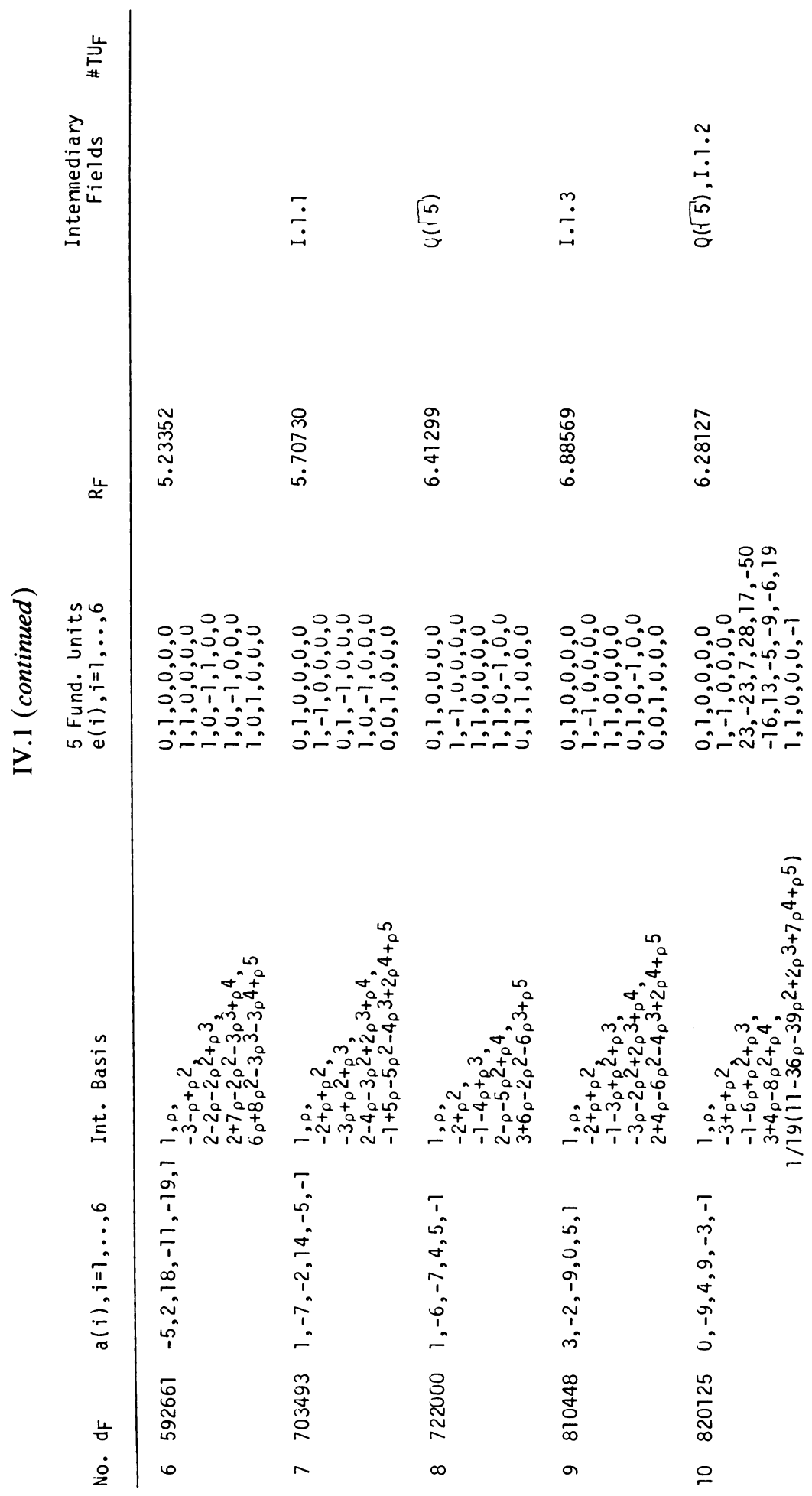




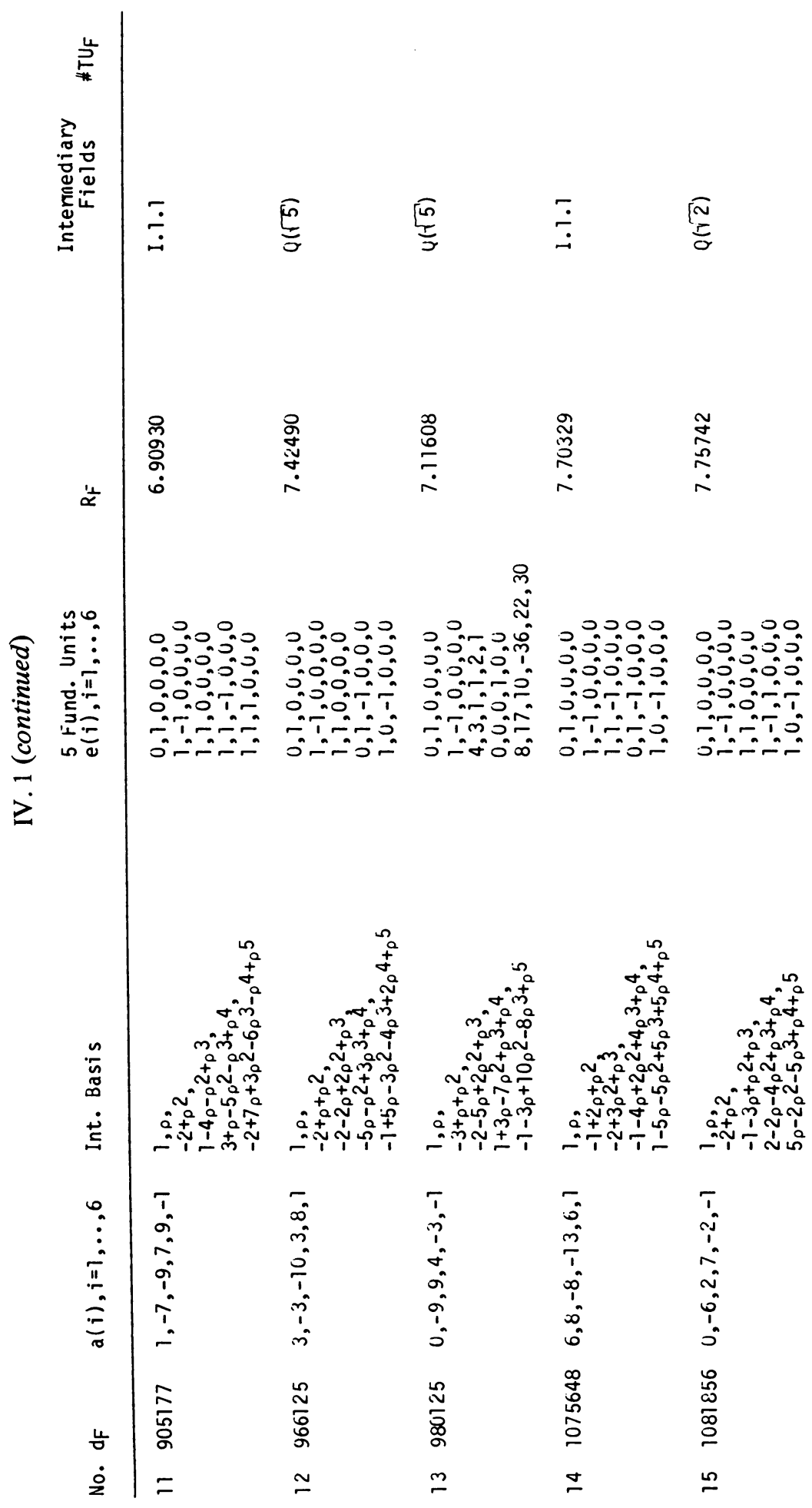




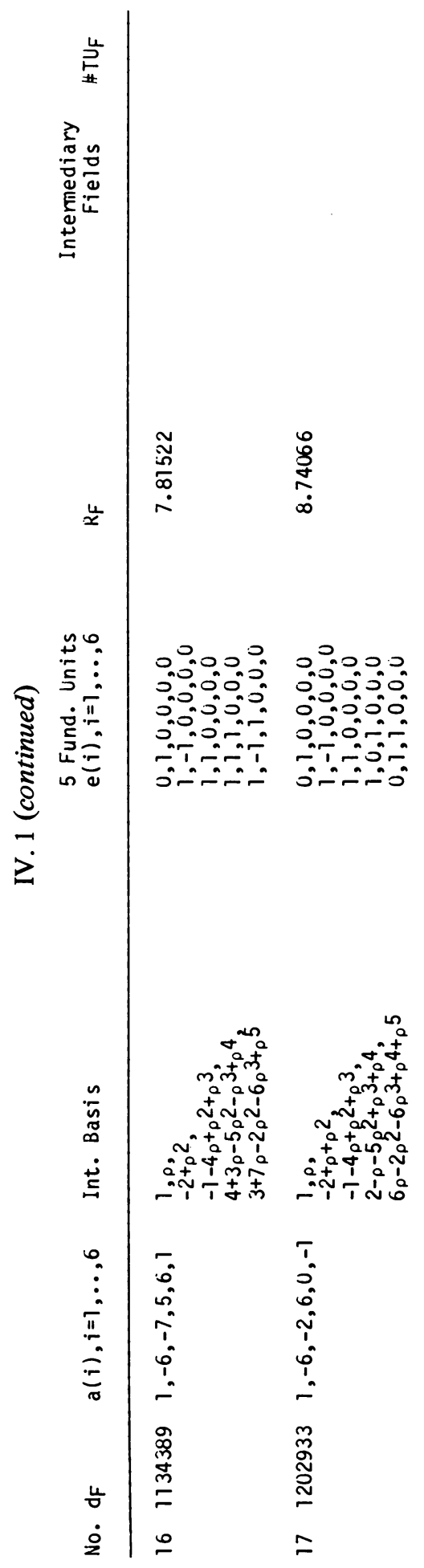



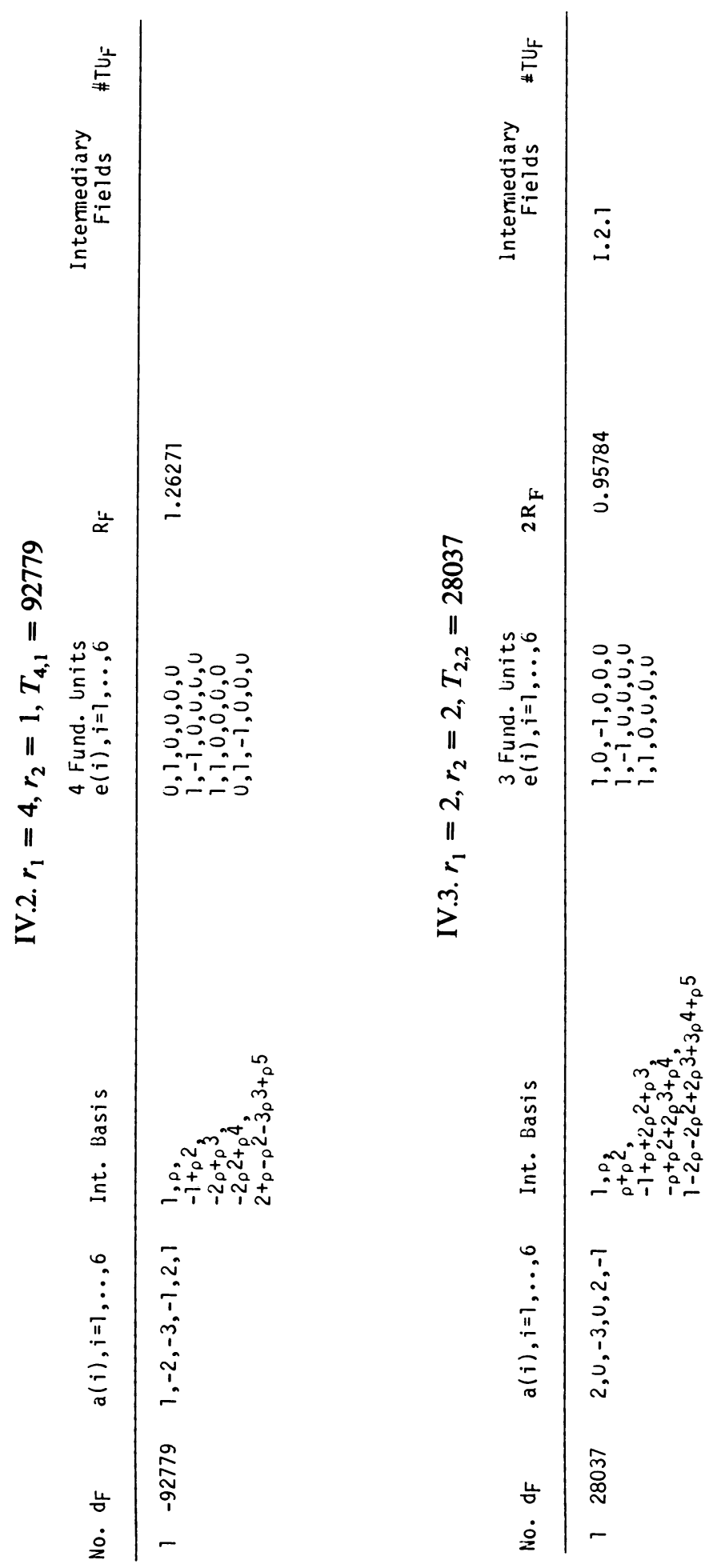


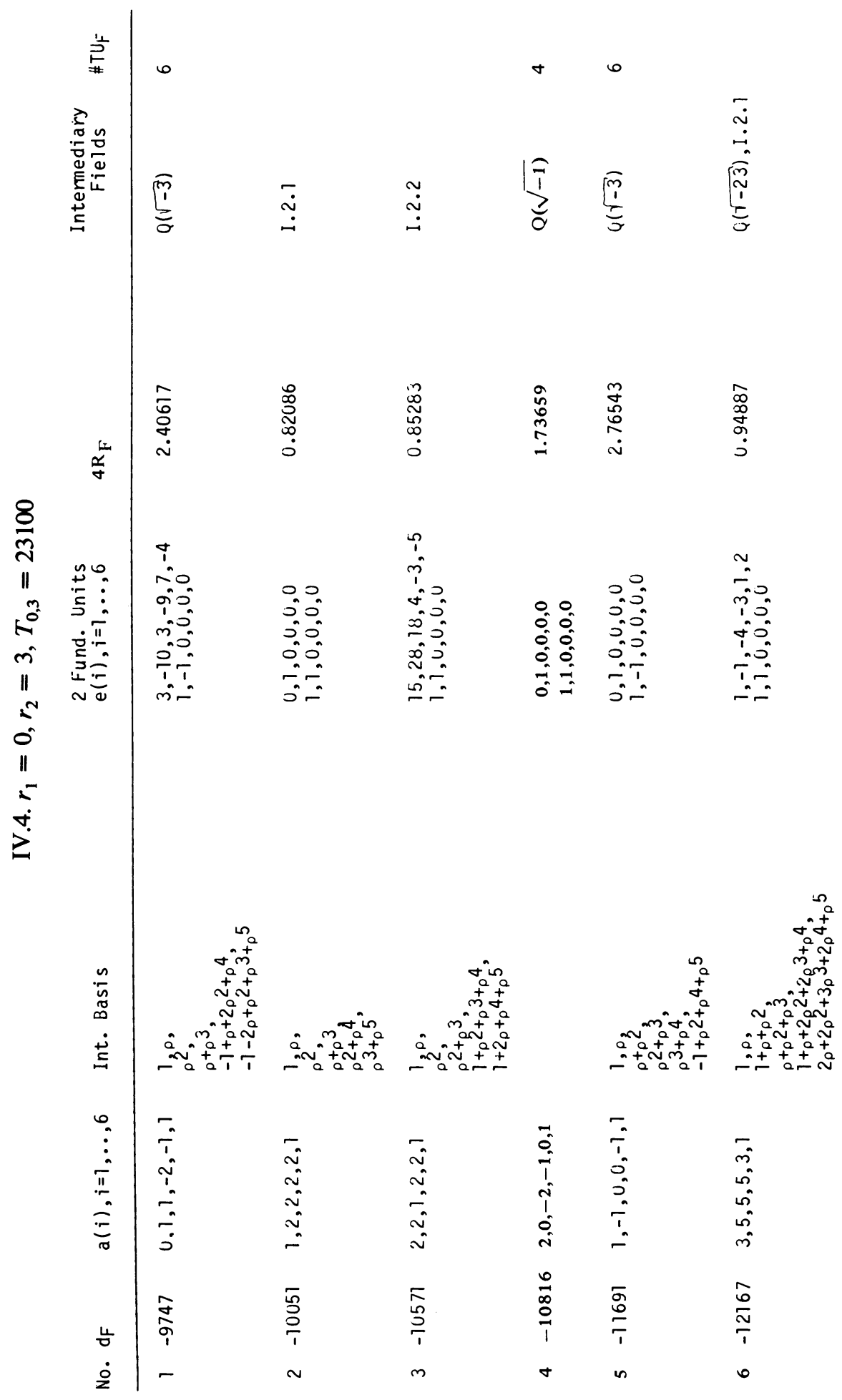




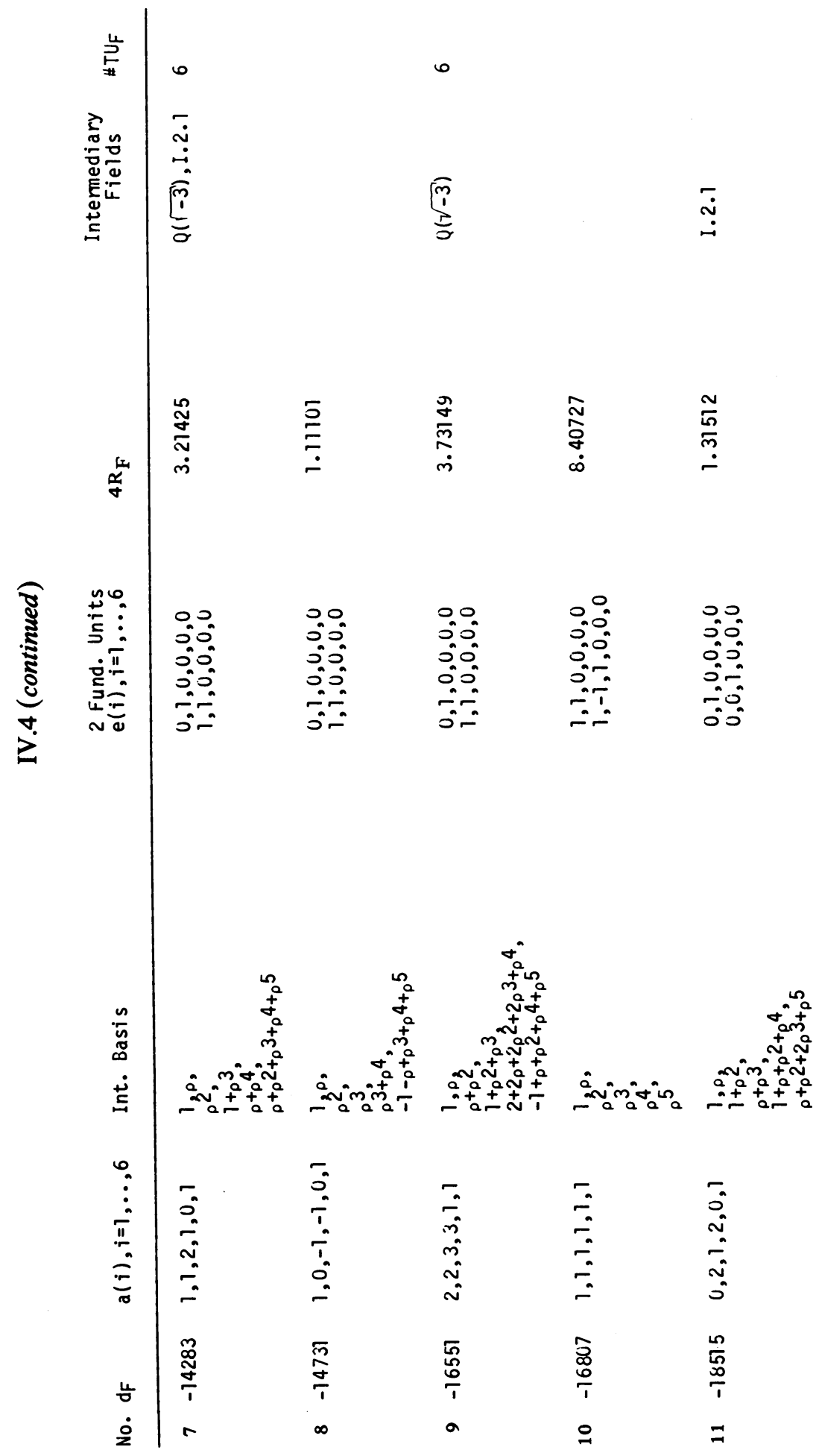




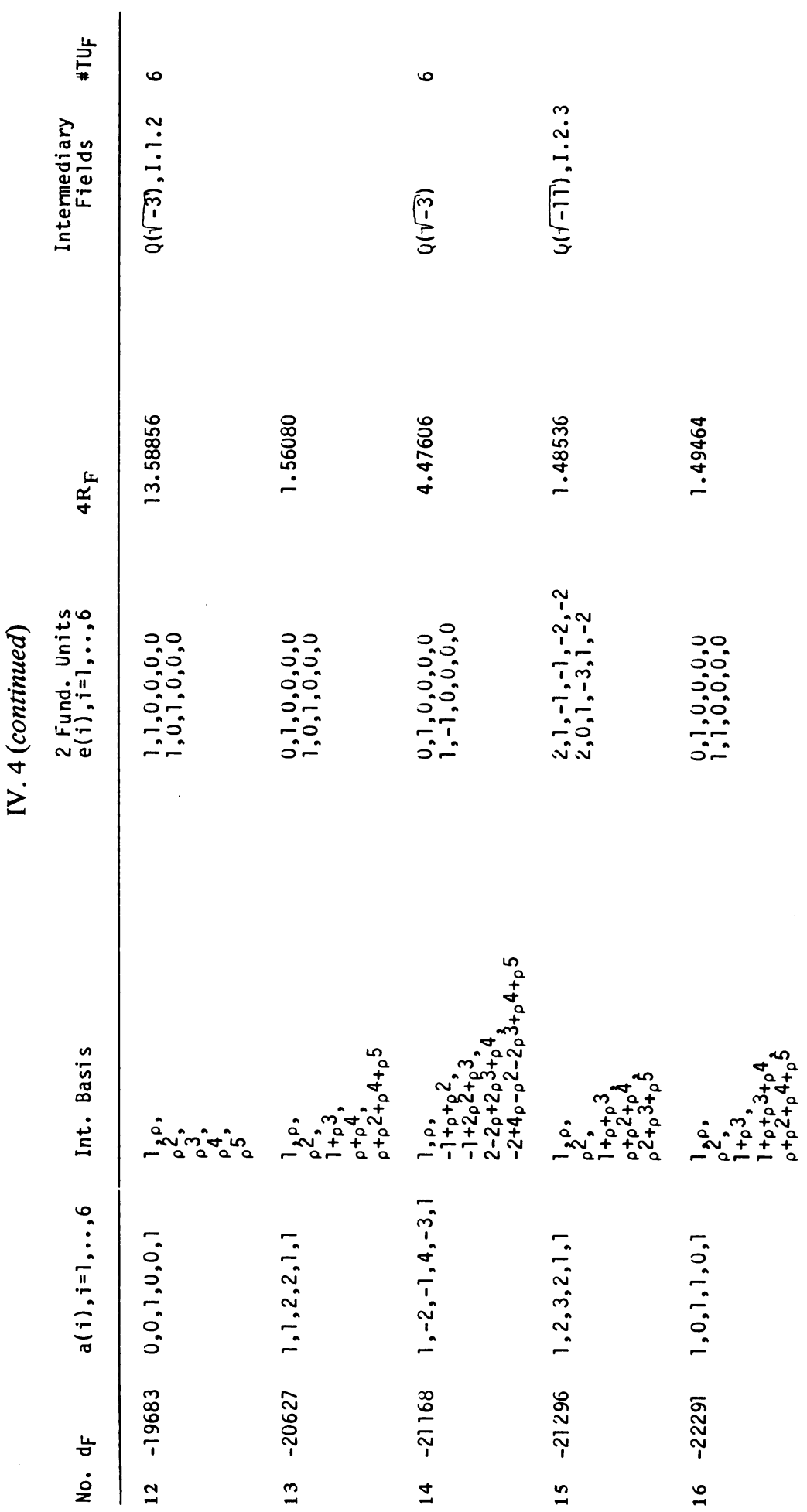




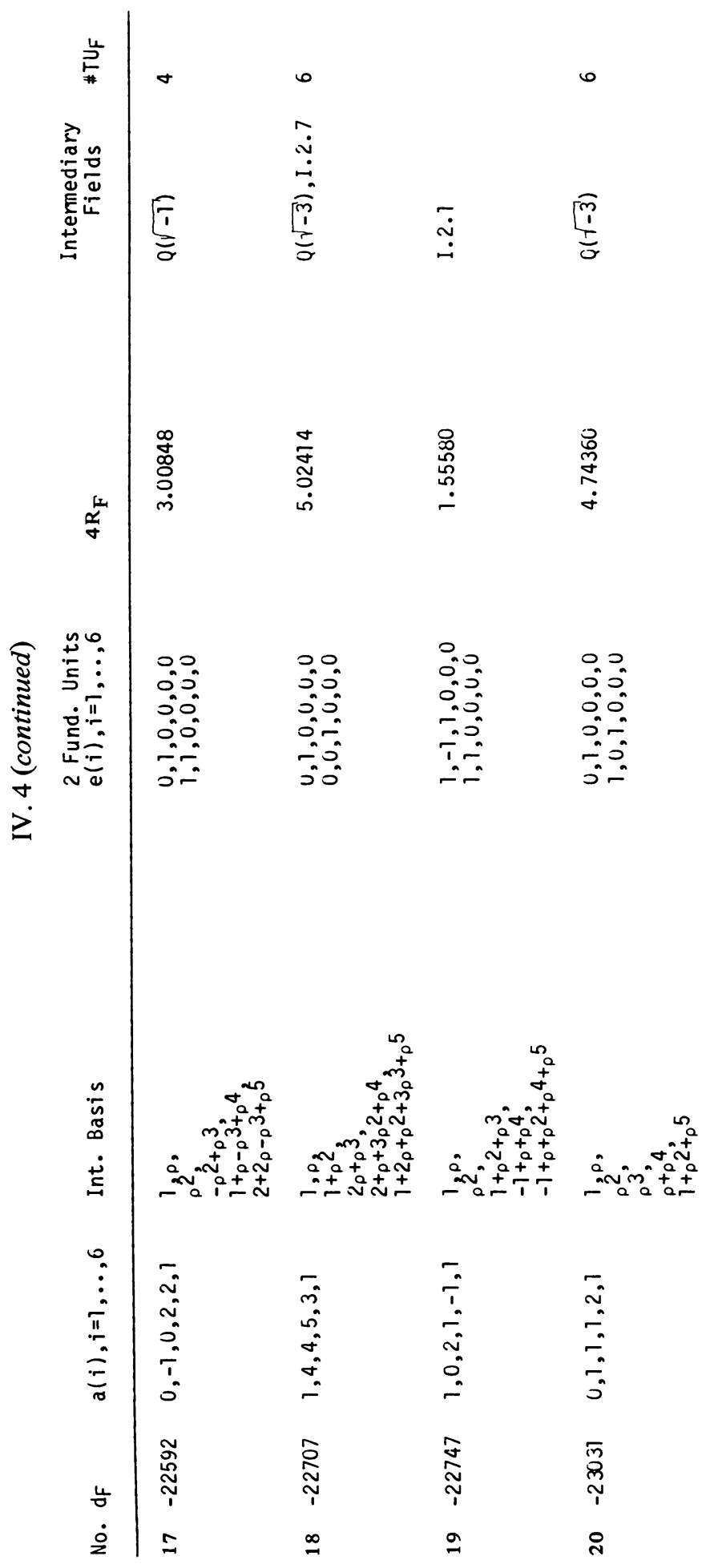


Mathematisches Institut

Universität Düsseldorf

Universitätsstr. 1

4 Düsseldorf, West Germany

Mathematisches Institut

Universität zu Köln

Weyertal 86-90

5 Köln 41, West Germany

Department of Mathematics

The Ohio State University

Columbus, Ohio 43210

1. D. Ford, On the Computation of the Maximal Order in a Dedekind Domain, Thesis, The Ohio State University, 1978.

2. M. PohST, "A program for determining fundamental units," in $S Y M S A C 76$, pp. 177-182.

3. М. PoHst, "On the computation of number fields of small discriminants including the minimum discriminants of sixth degree fields," J. Number Theory. (To appear.)

4. M. PонST, "On the computation of lattice vectors of minimal length, successive minima, and reduced bases with applications," ACM SIGSAM Bull., v. 15, 1981, pp. 37-44.

5. M. Pohst \& H. Zassenhaus, "An effective number geometric method of computing the fundamental units of an algebraic number field," Math. Comp., v. 31, 1977, pp. 754-770.

6. M. Pohst \& H. Zassenhaus, "On effective computation of fundamental units. I," Math. Comp., v. 38, 1982, pp. $275-291$. 